\title{
A Modified Triple-Diode Model Parameters Identification for Perovskite Solar Cells via Nature-Inspired Search Optimization Algorithms
}

\author{
Alaa A. Zaky ${ }^{1}(\mathbb{D})$, Ahmed Fathy ${ }^{2,3, *}$, Hegazy Rezk ${ }^{4}\left(\mathbb{D}\right.$, Konstantina Gkini ${ }^{5}\left(\mathbb{D}\right.$, Polycarpos Falaras $^{5}(\mathbb{D}$ \\ and Amlak Abaza ${ }^{1}$ \\ 1 Electrical Engineering Department, Kafrelsheikh University, Kafr El-Sheikh 33511, Egypt; \\ alaa.hussien@eng.kfs.edu.eg (A.A.Z.); amalk.elhariri@eng.kfs.edu.eg (A.A.) \\ 2 Electrical Engineering Department, Faculty of Engineering, Jouf University, Sakaka 72388, Saudi Arabia \\ 3 Electrical Power and Machines Department, Faculty of Engineering, Zagazig University, Zagazig 44511, Egypt \\ 4 Electrical Engineering Department, Faculty of Engineering, Minia University, Minia 61517, Egypt; \\ hegazy.hussien@mu.edu.eg \\ 5 National Centre for Scientific Research "Demokritos", Institute of Nanoscience and Nanotechnology, Agia \\ Paraskevi Attikis, 15341 Athens, Greece; k.gini@inn.demokritos.gr (K.G.); p.falaras@inn.demokritos.gr (P.F.) \\ * Correspondence: afali@ju.edu.sa
}

check for updates

Citation: Zaky, A.A.; Fathy, A.; Rezk, H.; Gkini, K.; Falaras, P.; Abaza, A. A Modified Triple-Diode Model Parameters Identification for Perovskite Solar Cells via Nature-Inspired Search Optimization Algorithms. Sustainability 2021, 13, 12969. https://doi.org/10.3390/ su132312969

Academic Editor:

Catherine Housecroft

Received: 25 October 2021

Accepted: 18 November 2021

Published: 23 November 2021

Publisher's Note: MDPI stays neutral with regard to jurisdictional claims in published maps and institutional affiliations.

Copyright: (c) 2021 by the authors. Licensee MDPI, Basel, Switzerland. This article is an open access article distributed under the terms and conditions of the Creative Commons Attribution (CC BY) license (https:// creativecommons.org/licenses/by/ $4.0 /)$.
Abstract: Recently, perovskite solar cells (PSCs) have been widely investigated as an efficient alternative for silicon solar cells. In this work, a proposed modified triple-diode model (MTDM) for PSCs modeling and simulation was used. The Bald Eagle Search (BES) algorithm, which is a novel nature-inspired search optimizer, was suggested for solving the model and estimating the PSCs device parameters because of the complex nature of determining the model parameters. Two PSC architectures, namely control and modified devices, were experimentally fabricated, characterized and tested in the lab. The I-V datasets of the fabricated devices were recorded at standard conditions. The decision variables in the proposed optimization process are the nine and ten unknown parameters of triple-diode model (TDM) and MTDM, respectively. The direct comparison with a number of modern optimization techniques including grey wolf (GWO), particle swarm (PSO) and moth flame (MFO) optimizers, as well as sine cosine (SCA) and slap swarm (SSA) algorithms, confirmed the superiority of the proposed BES approach, where the Root Mean Square Error (RMSE) objective function between the experimental data and estimated characteristics achieves the least value.

Keywords: perovskite solar cells; triple-diode model; optimization; energy efficiency

\section{Introduction}

Perovskite absorbers have been widely used in solar cells due to their suitable optoelectronic properties [1], such as high carrier mobility [2], tunable bandgap and long carrier lifetime [3]. Perovskite solar cells (PSCs) have demonstrated great power conversion efficiency (PCE) improvement in a very fast pace [4,5], which nowadays exceeds $25 \%$, overcoming other third-generation technologies [6]. The planar structure is characterized by ease and low-cost fabrication and consists of a compact electron transport layer (ETL), the absorber, a hole transport layer (HTL) and the metal contacts. Hysteresis phenomenon and stability issues are the most important drawbacks of planar PSCs. The functionality of the ETL, as well as its interface with the perovskite, are crucial for the overall PSCs performance and stability [7,8]. Several interface engineering approaches [9-12] have been implemented to confront the abovementioned issues. Recently, manganese-based porphyrin was incorporated in planar PSCs [13] as an electron transport mediator which facilitated the electron transfer, favored the growth of more homogenous perovskite films with larger and better crystallized grains and, thus, resulted in enhanced PCE and improved stability. 
There are three common models that have been developed to describe the solar cell: single-diode, double-diode, and triple-diode models. Such models depend on the diodes as their main components, along with some resistors to characterize the properties of the PV solar cell. However, the main issue that needs to be faced is finding the optimal values for individual parameters, such as impedance, diode ideal factors, and saturation currents, in order to obtain successful modeling. The single-diode model is considered the basic one and has a single diode. The development of a more detailed model would be more appropriate to represent a wide range of PV systems considering different conditions [14]. To increase the accuracy of conventional model, some modified models are developed. Dalia et al. [14] suggested an improved double-diode model. They added an extra resistor in series with the second diode to consider the influence of grain boundary regions. An improved triple-diode model was therein suggested and compared with the improved double-diode model. The results proved that the accuracy of an improved triple-diode model is better than an improved double-diode model [15].

In this paper, the modified TDM is examined to model the perovskite solar cells using the bald eagle search (BES) algorithm. Two perovskite-based devices, namely, control and modified, were experimentally fabricated, characterized and tested in the lab. Characteristic I-V parameters of the fabricated devices were recorded at standard condition. A comparison study is presented with other optimization algorithms of grey wolf optimizer (GWO), particle swarm optimizer (PSO), moth flame optimizer (MFO), (SCA) and (SSA), where the superiority of the proposed BES algorithm is proved.

The main contributions of this paper are summarized as follows:

- a new application of the bald eagle search algorithm to identify the parameters of the modified TDM for perovskite solar cells;

- the obtained results by the bald eagle search algorithm are compared with other methods;

- the accuracy and superiority of the bald eagle search algorithm in determining parameters of the modified TDM for perovskite solar cells are proved.

The rest of the paper is arranged as follows: Section 2 presents the details of the experimental work. The mathematical representation of the modified TDM and problem formulation is presented in Section 3. A brief overview of the bald eagle search algorithm is explained in Section 4. The obtained results are presented and discussed in Section 5. Finally, Section 6 outlines the main findings of the current research work.

\section{Experimental}

\subsection{Device Fabrication}

Fluorine-doped tin oxide (FTO) conductive substrates were cleaned thoroughly by sequential 15 min ultra-sonication baths with Hellmanex, 2-propanol, and acetone. Before the ETL's deposition, the FTOs underwent UV ozone treatment for $15 \mathrm{~min}$. For the compact layer, a solution of titanium (IV) isopropoxide in ethanol was spin-coated at $2000 \mathrm{rpm}$ for $60 \mathrm{~s}$ and the resulted $\mathrm{TiO}_{2}$ films were annealed at $500{ }^{\circ} \mathrm{C}$ for $45 \mathrm{~min}$ with a temperature ramp rate of $5{ }^{\circ} \mathrm{C} \mathrm{min}^{-1}$. For the modified films, manganese porphyrin was diluted in methanol, resulting in a solution of $0.5 \mathrm{mg} \mathrm{mL}^{-1}$, and was spin-coated at $2000 \mathrm{rpm}$ for $40 \mathrm{~s}$ on top of the $\mathrm{TiO}_{2}$ films. The perovskite solution and the HTM solution were prepared and deposited into an argon-filled glovebox. For the perovskite solution, $40 \mathrm{wt} \%$ of methylammonium iodide and lead acetate trihydrate were diluted in anhydrous DMF in a 3:1 molar ratio and a small amount of hypophosphorous acid (50\% $w / w$, aqueous solution) was added at the end. The perovskite solution was spin-coated on the ETLs at $2000 \mathrm{rpm}$ for $45 \mathrm{~s}$. The obtained films were left to dry at room temperature for $10 \mathrm{~min}$ and afterward annealed at $100{ }^{\circ} \mathrm{C}$ for $5 \mathrm{~min}$. After cooling down, a $70 \mathrm{mM}$ Spiro-MeOTAD solution in chlorobenzene, containing additives of lithium bis-(trifluoromethanesulfonyl) imide lithium salt in acetonitrile, 4-tert-butylpyridine and FK209 Co(III) TFSI salt in acetonitrile, was spin-coated at $4000 \mathrm{rpm}$ for $10 \mathrm{~s}$. Finally, $100 \mathrm{~nm}$ of patterned silver electrodes were thermally evaporated under a $10^{-6}$ Torr vacuum at a $\sim 1 \AA \mathrm{s}-1$ rate. Figure 1 Cross-section 
SEM image (Reprinted with permission from [13]. Copyright 2020 American Chemical Society) shows the schematic illustration, an optical image and a cross-section SEM image of the obtained devices.

a)

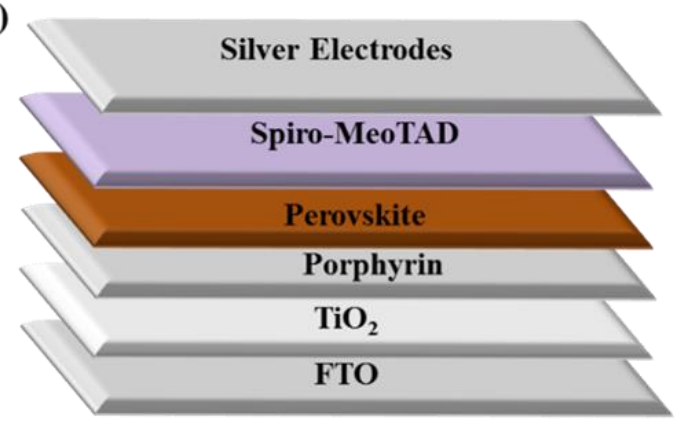

b)

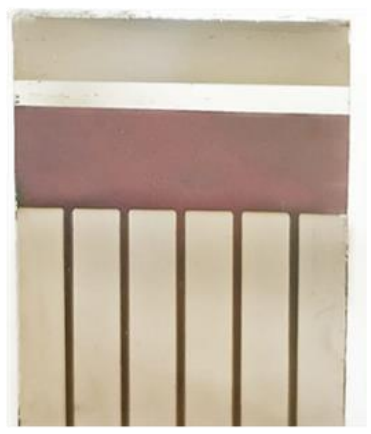

c)

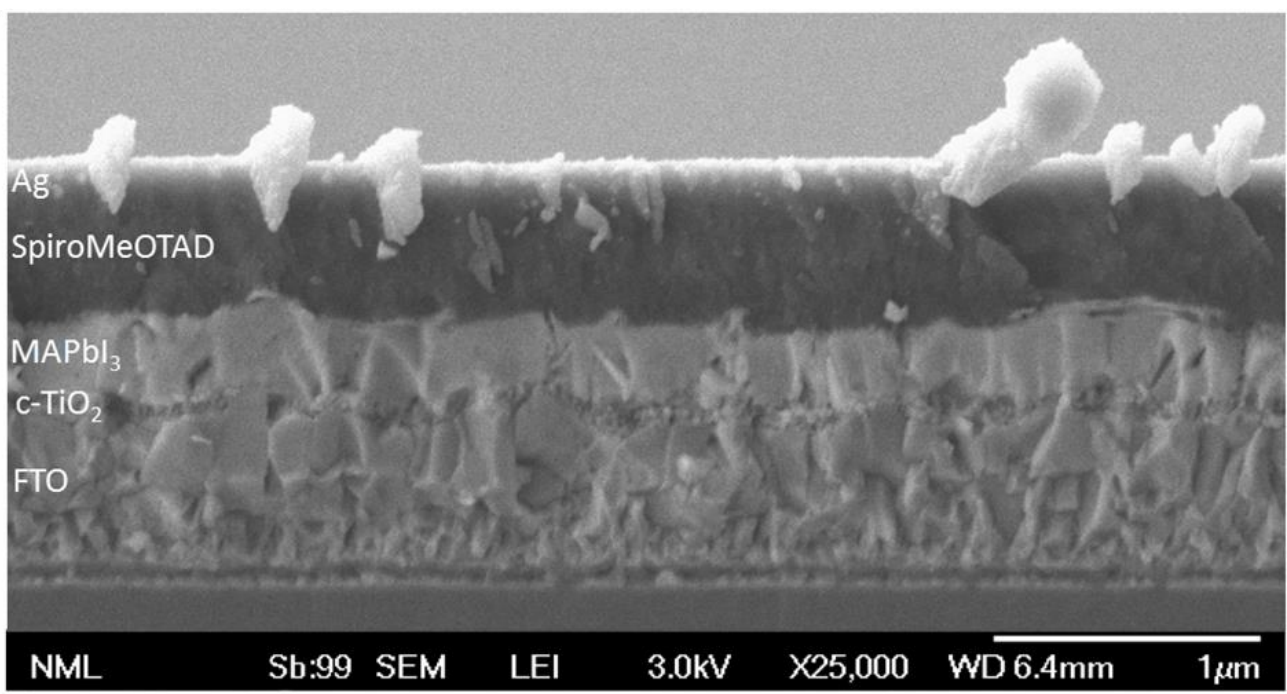

Figure 1. (a) Schematic representation of the PSC architecture. (b) The fabricated PSC device overview. (c) The cross-section SEM image of the fabricated devices.

\subsection{Characterization Methods}

The devices were characterized electrochemically using a solar simulator (Solar Light Company, Inc. (Glenside, PA, USA) 300 W model 16 S-300/1 sun, AM 1.5 D, $1000 \mathrm{~W} \mathrm{~m}^{-2}$ ) as the illumination source and an Autolab PG-STAT-30 potentiostat to obtain the current-voltage $(\mathrm{J}-\mathrm{V})$ curves with a scan rate of $50 \mathrm{mV} \mathrm{s}^{-1}$. In addition, electrochemical impedance spectroscopy (EIS) measurements were carried out under dark and 1 sun illumination conditions using the PG-STAT-30 potentiostat and its built-in frequency response analyzer (Figure 2). The UV-vis absorption spectra were recorded with a PerkinElmer LAMBDA $40 \mathrm{UV}$ - vis spectrometer. In [13], all the details about the fabricated devices performance are discussed. 


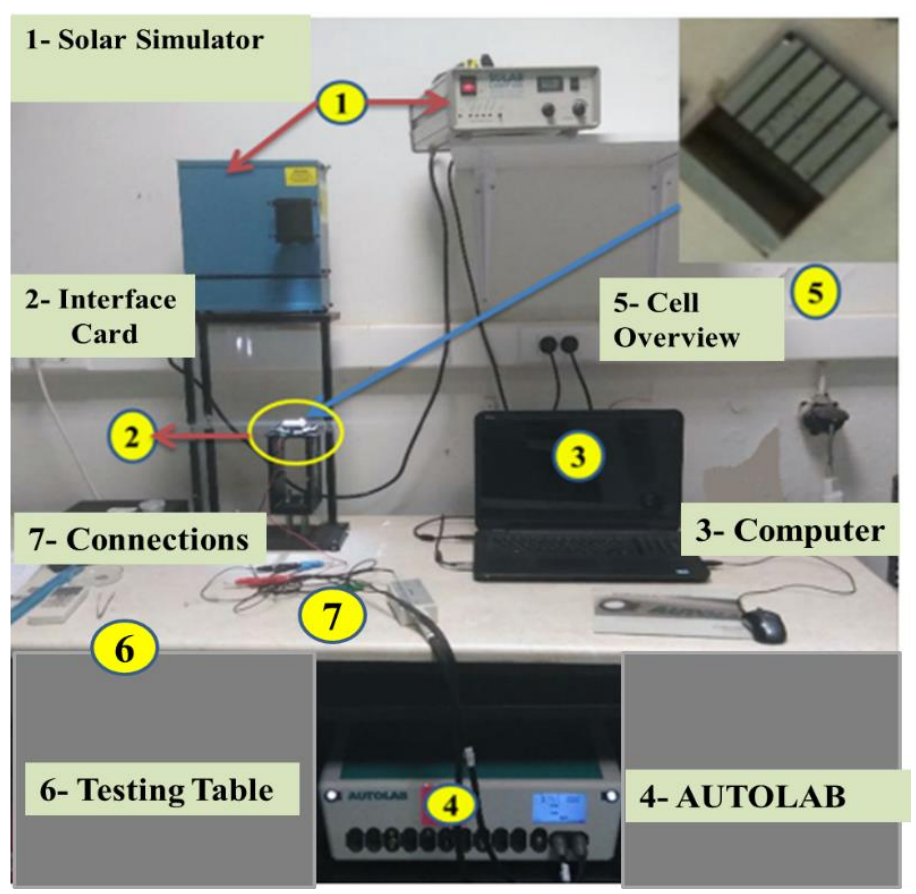

Figure 2. Laboratory experimental testing board for PSC device testing.

\section{Triple-Diode Model and Problem Formulation}

Figure 3 shows the TDM equivalent circuit, while Figure 4 shows the modified TDM equivalent circuit of PV. The mathematical model of both TDM and modified TDM are presented in this section.

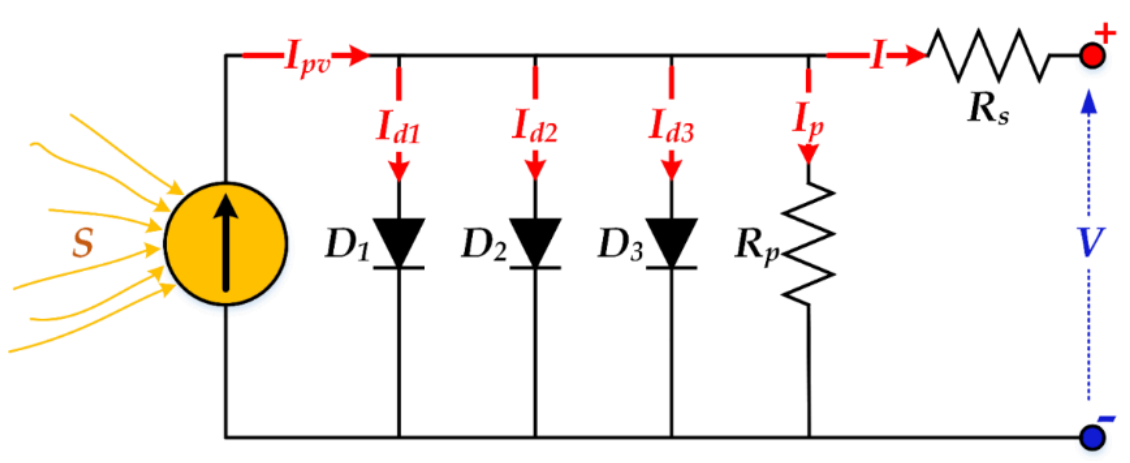

Figure 3. Equivalent circuit of TDM of PV solar cell.

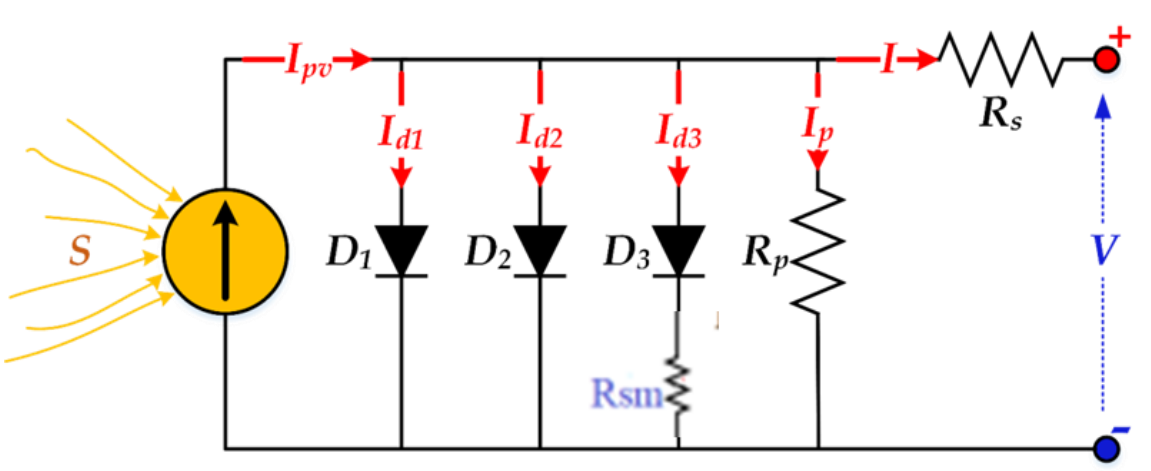

Figure 4. Equivalent circuit of modified TDM of PV solar cell. 
From the equivalent circuit shown in Figure 3, the solar cell output current is represented by the following equation [14].

$$
I=I_{p v}-I_{D 1}-I_{D 2}-I_{D 3}-\left(\frac{V+I \cdot R_{s}}{R_{p}}\right)
$$

where $I_{p v}$ denotes the photo-generated current. $I_{D 1}, I_{D 2}, I_{D 3}$ denote the current in the first, second and third diodes, respectively. $R_{s}$ is the series resistance. $R_{p}$ denotes the shunt resistance.

The diodes currents can be estimated as shown in Equation (2), based on the Shockley formula as follows:

$$
\begin{aligned}
& I_{D 1}=I_{01}\left(e^{\left(\frac{V+I \cdot R_{s}}{n_{1} \cdot V_{t}}\right)}-1\right) \\
& I_{D 2}=I_{02}\left(e^{\left(\frac{V+I . R_{s}}{n_{2} \cdot V_{t}}\right)}-1\right) \\
& I_{D 3}=I_{03}\left(e^{\left(\frac{V+I . R_{s}}{n_{3} \cdot V_{t}}\right)}-1\right)
\end{aligned}
$$

where $n_{1}, n_{2}$, and $n_{3}$ denote the ideality factor of $D_{1}, D_{2}$, and $D_{3}$, respectively; $I_{01}, I_{02}$ and $I_{03}$ are the saturation currents of diodes $D_{1}, D_{2}$, and $D_{3}$, respectively. $V_{t}$ denotes the thermal voltage. It can be calculated using the following relation.

$$
V_{t}=\frac{k T N_{s}}{q}
$$

where $k$ denotes the Boltzmann constant, $T$ denotes the temperature of the PV panel, $N_{s}$ is the number of series solar cells, and $q$ denotes the electron charge.

Based on equivalent circuit shown in Figure 3, the solar cell output current using the TDM can be obtained from the following relation [14].

$$
I=I_{p v}-I_{01}\left(e^{\left(\frac{V+I \cdot R_{s}}{n_{1} \cdot V_{t}}\right)}-1\right)-I_{02}\left(e^{\left(\frac{V+I \cdot R_{s}}{n_{2} \cdot V_{t}}\right)}-1\right)-I_{03}\left(e^{\left(\frac{V+I \cdot R_{s}}{n_{3} \cdot V_{t}}\right)}-1\right)-\frac{V+I \cdot R_{s}}{R_{p}}
$$

To increase the accuracy of the equivalent model, the modified TDM is proposed, where the losses in the defect region are included in the model and expressed via adding the modified series resistance; $R_{s m}$ in the third diode branch as shown in Figure 4, and Equation (4) updated to be as shown in Equation (5):

$$
I=I_{p v}-I_{01}\left(e^{\left(\frac{V+I . R_{s}}{n_{1} \cdot V_{t}}\right)}-1\right)-I_{02}\left(e^{\left(\frac{V+I . R_{s}}{n_{2} \cdot V_{t}}\right)}-1\right)-I_{03}\left(e^{\left(\frac{V+I . R_{s}-R_{s m} I_{d 3}}{n_{3} \cdot V_{t}}\right)}-1\right)-\frac{V+I \cdot R_{s}}{R_{p}}
$$

Coidering Equation (5), the modified TDM model contains ten unknown parameters $\left(I_{p v}, I_{01}, I_{02}, I_{03}, n_{1}, n_{2}, n_{3}, R_{s}, R_{p}, R_{s m}\right)$.

The ten unknown parameters of the modified TDM should be estimated accurately. An optimization process is used for obtaining the module parameters, where the root mean square error (RMSE) between the estimated and experimental data is used as an objective function. The RMSE is estimated using the following relation and should be minimum.

$$
R M S E=\sqrt{\frac{1}{N} \sum_{i=1}^{N}\left(I_{m}-I_{e}\right)^{2}}
$$

where $N$ denotes the number of datasets, $I_{m}$ denotes the measured current, and $I_{e}$ denotes the estimated current.

\section{Bald Eagle Search Optimization Algorithm}

Bald Eagle Search (BES) is a novel nature-inspired search optimizer. It is a metaheuristic algorithm based on applying intelligent strategies to hunt the fish. The bald eagle depends on intelligent social behavior to reach the best goal based on three stages. The first stage is the selection of the hunting area which contains the greatest number of fish. Searching in the hunting area is the second stage. In this stage, the eagle moves inside 
the space to search for fish by identifying the best position to hunt the prey. In the third stage, the eagle uses the position selected from the second stage and determines the best point to hunt. This stage is known as swooping. Swooping begins from the best point to hunt and all other movements are directed to this point. Figure 5 describes the three stages of BES [16].

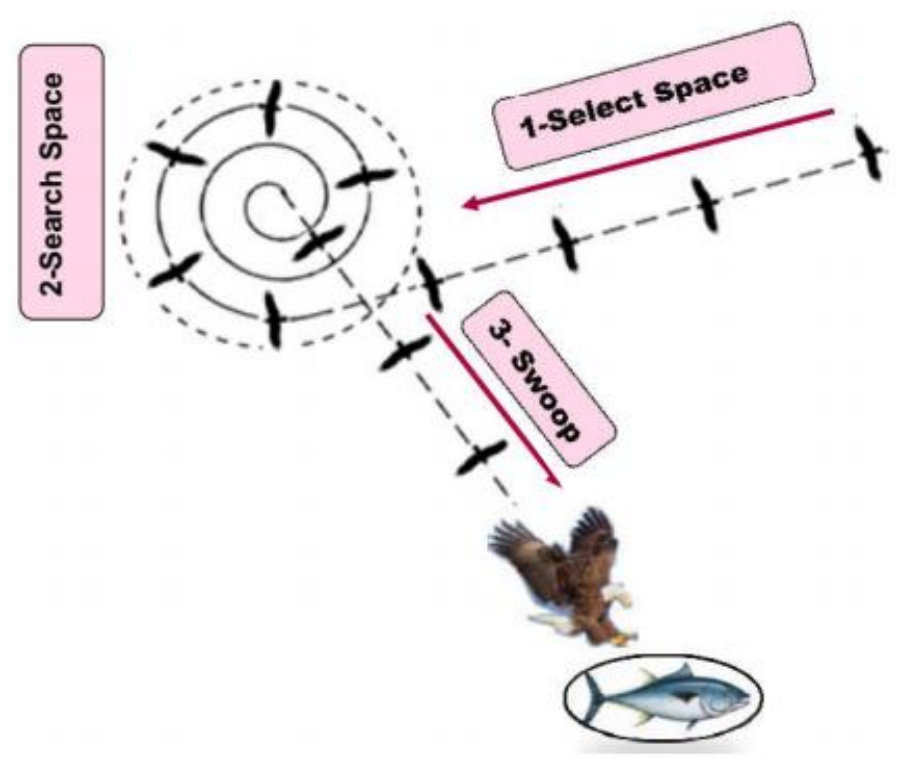

Figure 5. Bald Eagle Search Optimization Algorithm hunting strategy.

The considered hunting stages are formulated as follows:

\subsection{1st Stage: Select Stage}

In the select stage, eagles select the best hunting area depending on the amount of food there. Eagles use their previous search information about the hunting areas. The selecting behavior can be formulated as:

$$
P_{i, \text { new }}=P_{\text {best }}+\alpha \times r\left(P_{\text {mean }}-P_{i}\right)
$$

where $P_{\text {best }}$ represents the best hunting position determined from previous searches, where $\alpha$ is a controlling parameter for different best positions and it takes a value between 1.5 and 2. The random number, $r$, lies between 0 and $1 . P_{\text {mean }}$ refers to using all information from the previous search points. It is clear from Equation (7) that eagles randomly search for a new best space in all points near the previous selected search areas. The current movement from the previous best point changes randomly by multiplying the difference of search and mean points by the controlling parameter, $\alpha$.

\subsection{2nd Stage: Search Stage}

After selecting the search area, eagles move spirally in different directions within the selected search space, as shown in Figure 6. The best position of an eagle for the swoop is represented mathematically as:

$$
P_{i, \text { new }}=P_{i}+y(i) \times\left(P_{i}-P_{i+1}\right)+x(i) \times\left(P_{\text {mean }}-P_{i}\right)
$$

where,

$$
\begin{gathered}
x(i)=\frac{x r(i)}{\max (|x r|)}, y(i)=\frac{y r(i)}{\max (|y r|)} \\
x r(i)=r(i) \times \sin (\theta(i)), y r(i)=r(i) \times \cos (\theta(i)) \\
\theta(i)=a \times \pi \times \text { rand } \ldots \ldots
\end{gathered}
$$




$$
r(i)=\theta(i)+R \times \text { rand }
$$

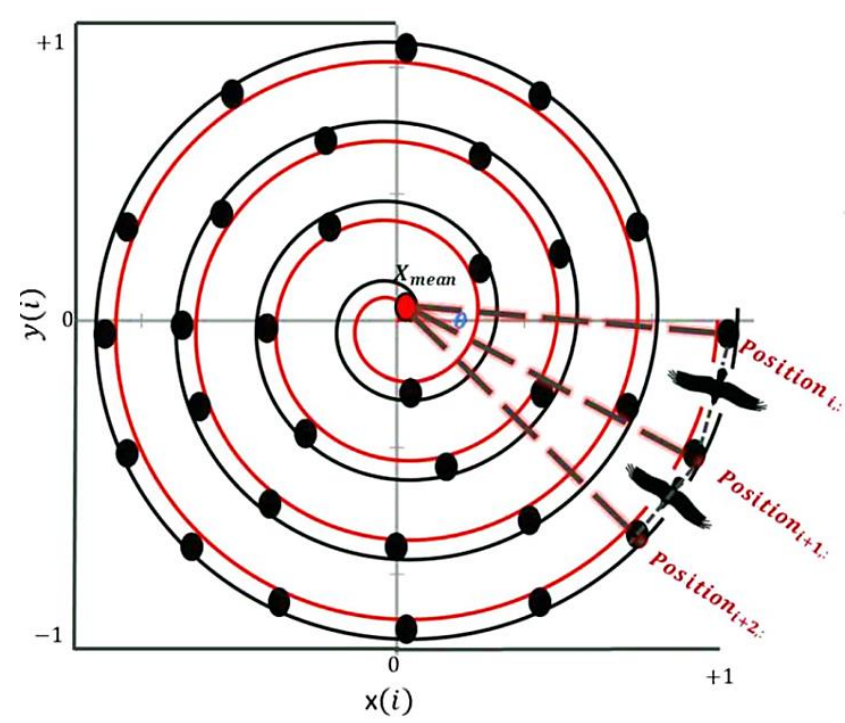

Figure 6. Searching in spiral space.

Equation (8) is explained using a set of equations, (9a) to (9d), and using the shape of spiral movement. The parameters a and $R$ determine the number of cycles and the shape of the movement. The value of a varies from 5 to 10, while $R$ takes values in between 0.5 and 2. The movement of the eagle towards the center point is represented in polar axes. The updating of current position is considered by adding two parts in the y-axis and the $\mathrm{x}$ - axis. The first adding part is the multiplying of the point position of the polar in the $y$-axis e by the difference between the current and next point. The second part adding to the current point is the point position of the polar in the x-axis multiplying by the difference between the current point and the mean point. The spiral movement of eagles from one point to another represents the search space and all points move towards the center point. All position of points in the polar plots lies between 1 and -1 . The best point to swoop is determined compared to the center point.

\subsection{3rd Stage: Swooping Stage}

Once the eagles determine the best point to swoop in the search space, they move in high speed towards their focused prey. The eagles move from the center point of search space towards the prey. The updating of the position of all points depends on the best location and the mean point, as follows:

$$
\begin{gathered}
P_{i, \text { new }}=\text { rand } \times P_{\text {best }}+x 1(i) \times\left(P_{i}-c 1 \times P_{\text {mean }}\right)+y 1(i) \times\left(P_{i}-c 2 \times P_{\text {best }}\right) \\
x 1(i)=\frac{x r(i)}{\max (|x r|)}, y 1(i)=\frac{y r(i)}{\max (|y r|)} \\
x r(i)=r(i) \times \sinh (\theta(i)), y r(i)=r(i) \times \cosh (\theta(i)) \\
\theta(i)=a \times \pi \times \text { rand } \ldots \ldots \text { and } r(i)=\theta(i)
\end{gathered}
$$

where $c 1$ and $c 2 \in[1,2]$. These parameters result in increasing the movement intensity towards the prey. The whole optimization process steps are shown in Figure 7. 


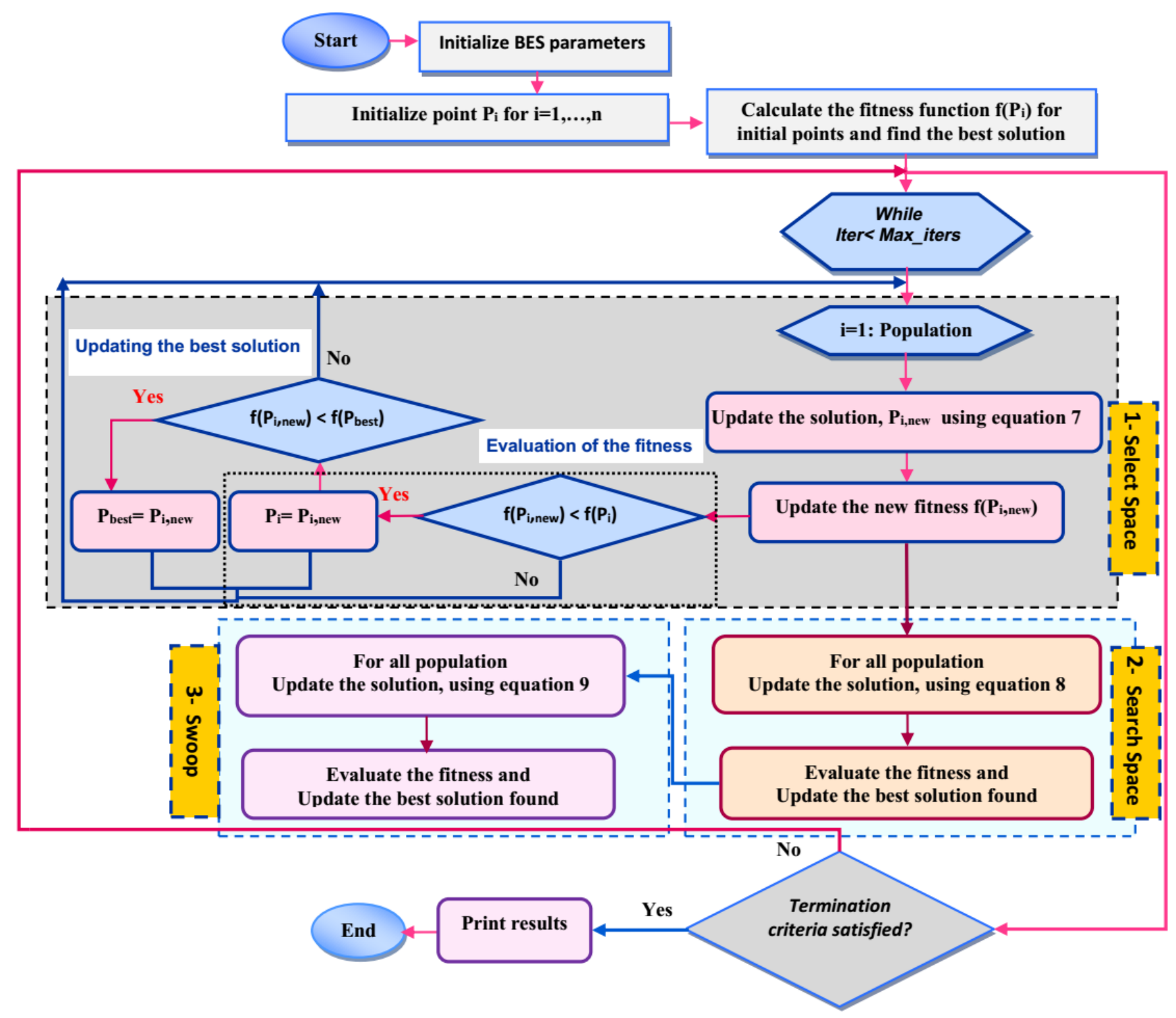

Figure 7. Optimization process using BES.

\section{Results and Discussion}

The absorption spectra (not shown) of the perovskite deposited on pristine and modified TiO2 confirmed that the ETL modification did not significantly affect the optical properties of the perovskite absorber [13].

The photovoltaic parameters of the PSCs including the short circuit current density (Jsc), the open circuit voltage (Voc), the fill factor (FF) and the power conversion efficiency (PCE) were determined for both control and modified devices under 1 sun illumination conditions [13]. The modified PSCs demonstrated significantly higher photovoltaic performance reaching power conversion efficiency (PCE) of $18.7 \%$, clearly outperforming that of the control device $(16.9 \%)$. This was attributed to better crystalized and more homogenous perovskite films $[17,18]$, associated with a smoother and more functional ETL/absorber interface [19-34]. The PCE enhancement for the modified devices was associated with the increase of $\mathrm{J}_{\mathrm{sc}}$ and FF parameters, while the $\mathrm{V}_{\mathrm{oc}}$ value was almost the same $(\sim 1.04 \mathrm{~V})$. Specifically, the best-performing modified PSC provided a short circuit current density of $23.52 \mathrm{~mA} \mathrm{~cm}^{-2}$, which was $4 \%$ larger than that of the reference one. The observed increase was assigned to the presence of the porphyrin's negative dipole moment at the interface, which facilitated the electrons transfer to the photoanode. An additional improvement was 
registered in the case of fill factor, where the modified device presented a higher FF value (0.75) compared to the reference one (0.71).

A techno-economic study for the third-generation PSCs in real water pumping system application for irrigation purpose was carried out in [34], proving the superiority of the PSCs in comparison with the silicon-based technology solar cells where, for the same application, for the PSCsbased case there is a cost-saving and it needs only $3.42 \mathrm{~m}^{2}$, as the installation area, which is very small in comparison with the corresponding area of conventional silicon-based solar cells that exceeds $36 \mathrm{~m}^{2}$.

In the current study, the PSCs' parameters estimation based on TDM and MTDM was made via BES algorithm to prove the enhanced efficiency and precision of the proposed algorithm.

Two PSC devices, namely, control and modified devices experimental data, have been used in this work. The devices were fabricated as explained in the experimental section, while the photovoltaic performance was tested at standard condition $\left(25^{\circ} \mathrm{C}, 1000 \mathrm{~W} / \mathrm{m}^{2}\right)$.

The population size and number of iterations are the same for all optimization algorithms used in this study for a fair comparison. The least RMSE between the experimental data and the estimated current density of PSCs is the main purpose of the optimization procedure in this study. The parameters of both TDM and MTDM are the decision variables in the used optimization procedure. Tables 1 and 2 summarize the best parameters of the PSC devices based on TDM and MTDM, estimated by different optimization algorithms besides the lower and upper limits of the PSC device parameters. For better evaluation of the proposed BES algorithm performance, the studied optimizers are implemented 30 times. Statistical analysis for the optimization algorithms used in this study is shown in Tables 3 and 4. The details of 30 Runs for the control and modified PSCs recorded with TDM and MTDM are presented in Tables 5-8. From Tables 1 and 2, the estimated parameters agree with experimental ones with minor error values, while the best results are obtained by using both the proposed MTDM and the BES algorithm. For PSCs performance better understanding, parameters like diode ideality factors and diodes saturation currents values are very important but difficult to be determined experimentally. An important advantage of the simulation models (TDM, MTDM) is the ability to easily determine these parameters. Thus, the analysis and performance evaluation of the PSCs is further supported.

Table 1. Optimal parameters of PSCs applying different optimizers using TDM.

\begin{tabular}{|c|c|c|c|c|c|c|c|c|}
\hline \multirow{2}{*}{ Parameter } & \multicolumn{2}{|c|}{ Boundary } & \multicolumn{6}{|c|}{ Optimal Parameters } \\
\hline & Lower & Upper & BES & GWO & PSO & MFO & SCA & SSA \\
\hline \multicolumn{9}{|c|}{ Control Device } \\
\hline$I_{s c}(\mathrm{~A})$ & $1.0 \times 10^{-3}$ & $5.0 \times 10^{-3}$ & $2.2660 \times 10^{-3}$ & $2.2660 \times 10^{-3}$ & $2.2660 \times 10^{-3}$ & $2.2660 \times 10^{-3}$ & $2.2660 \times 10^{-3}$ & $2.2604 \times 10^{-3}$ \\
\hline$I_{\mathrm{O} 1}(\mathrm{~A})$ & $1.0 \times 10^{-20}$ & $5.0 \times 10^{-5}$ & $2.20 \times 10^{-20}$ & $1.09 \times 10^{-20}$ & $1.00 \times 10^{-20}$ & $1.00 \times 10^{-20}$ & $7.49 \times 10^{-20}$ & $1.20 \times 10^{-9}$ \\
\hline$I_{\mathrm{O} 2}(\mathrm{~A})$ & $1.0 \times 10^{-20}$ & $5.0 \times 10^{-5}$ & $1.00 \times 10^{-20}$ & $1.24 \times 10^{-20}$ & $1.00 \times 10^{-20}$ & $3.07 \times 10^{-10}$ & $7.40 \times 10^{-20}$ & $1.00 \times 10^{-20}$ \\
\hline$I_{\mathrm{O} 3}(\mathrm{~A})$ & $1.0 \times 10^{-20}$ & $5.0 \times 10^{-5}$ & $3.04 \times 10^{-10}$ & $2.63 \times 10^{-10}$ & $7.17 \times 10^{-11}$ & $1.00 \times 10^{-20}$ & $1.47 \times 10^{-9}$ & $2.65 \times 10^{-9}$ \\
\hline$n_{1}$ & 1.0 & 3.0 & 2.986 & 2.217 & 3.000 & 1.067 & 1.358 & 2.932 \\
\hline$n_{2}$ & 1.0 & 5.0 & 1.067 & 1.075 & 5.000 & 2.625 & 1.374 & 1.044 \\
\hline$n_{3}$ & 1.0 & 5.0 & 2.623 & 2.596 & 2.376 & 5.000 & 2.904 & 4.894 \\
\hline$R_{P}$ & 1000 & 7000 & 7000.00 & 6990.46 & 6997.79 & 7000.00 & 7000.00 & 6926.41 \\
\hline$R_{S}$ & 5.00 & 19.00 & 18.00 & 18.07 & 18.00 & 18.00 & 18.00 & 18.89 \\
\hline RMSE & & & $1.379 \times 10^{-5}$ & $1.397 \times 10^{-5}$ & $1.564 \times 10^{-5}$ & $1.379 \times 10^{-5}$ & $3.926 \times 10^{-5}$ & $2.243 \times 10^{-5}$ \\
\hline \multicolumn{9}{|c|}{ Modified Device } \\
\hline$I_{\mathrm{sc}}(\mathrm{A})$ & $1.0 \times 10^{-3}$ & $5.0 \times 10^{-3}$ & $2.455 \times 10^{-3}$ & $2.459 \times 10^{-3}$ & $2.464 \times 10^{-3}$ & $2.458 \times 10^{-3}$ & $2.554 \times 10^{-3}$ & $2.496 \times 10^{-3}$ \\
\hline$I_{\mathrm{o} 1}(\mathrm{~A})$ & $1.0 \times 10^{-20}$ & $5.0 \times 10^{-5}$ & $4.519 \times 10^{-11}$ & $3.800 \times 10^{-10}$ & $1.000 \times 10^{-20}$ & $1.000 \times 10^{-20}$ & $1.303 \times 10^{-20}$ & $1.000 \times 10^{-20}$ \\
\hline$I_{\mathrm{o} 2}(\mathrm{~A})$ & $1.0 \times 10^{-20}$ & $5.0 \times 10^{-5}$ & $2.094 \times 10^{-20}$ & $4.595 \times 10^{-10}$ & $4.064 \times 10^{-9}$ & $1.987 \times 10^{-10}$ & $3.547 \times 10^{-20}$ & $7.653 \times 10^{-9}$ \\
\hline$I_{03}(\mathrm{~A})$ & $1.0 \times 10^{-20}$ & $5.0 \times 10^{-5}$ & $6.332 \times 10^{-20}$ & $8.189 \times 10^{-10}$ & $1.000 \times 10^{-20}$ & $1.000 \times 10^{-20}$ & $4.103 \times 10^{-7}$ & $9.819 \times 10^{-14}$ \\
\hline$n_{1}$ & 1.0 & 3.0 & 2.280 & 2.592 & 3.000 & 3.000 & 1.468 & 1.035 \\
\hline$n_{2}$ & 1.0 & 5.0 & 4.982 & 3.994 & 3.056 & 2.509 & 1.055 & 3.336 \\
\hline$n_{3}$ & 1.0 & 5.0 & 4.967 & 4.352 & 5.000 & 1.065 & 5.000 & 2.112 \\
\hline$R_{P}$ & 1000 & 9000 & 9000.000 & 8976.499 & 9000.000 & 9000.000 & 5083.609 & 5194.242 \\
\hline$R_{S}$ & 5.000 & 17.50 & 17.000 & 11.941 & 5.000 & 17.000 & 5.153 & 14.262 \\
\hline RMSE & & & $1.632 \times 10^{-5}$ & $2.033 \times 10^{-5}$ & $3.006 \times 10^{-5}$ & $1.857 \times 10^{-5}$ & $1.013 \times 10^{-4}$ & $5.039 \times 10^{-5}$ \\
\hline
\end{tabular}


Table 2. Optimal parameters of PSCs applying different optimizers using MTDM.

\begin{tabular}{|c|c|c|c|c|c|c|c|c|}
\hline \multirow{2}{*}{ Parameter } & \multicolumn{2}{|c|}{ Boundary } & \multicolumn{6}{|c|}{ Optimal Parameters } \\
\hline & Lower & Upper & BES & GWO & POS & MFO & SCA & SSA \\
\hline \multicolumn{9}{|c|}{ Control Device } \\
\hline$I_{s c}(\mathrm{~A})$ & $1.0 \times 10^{-3}$ & $3.0 \times 10^{-3}$ & $2.266 \times 10^{-3}$ & $2.266 \times 10^{-3}$ & $2.266 \times 10^{-3}$ & $2.266 \times 10^{-3}$ & $2.266 \times 10^{-3}$ & $2.264 \times 10^{-3}$ \\
\hline$I_{\mathrm{O} 1}(\mathrm{~A})$ & $1.0 \times 10^{-20}$ & $5.0 \times 10^{-5}$ & $3.037 \times 10^{-10}$ & $2.447 \times 10^{-20}$ & $1.000 \times 10^{-20}$ & $3.242 \times 10^{-10}$ & $1.000 \times 10^{-20}$ & $2.452 \times 10^{-10}$ \\
\hline$I_{\mathrm{O} 2}(\mathrm{~A})$ & $1.0 \times 10^{-20}$ & $5.0 \times 10^{-5}$ & $1.000 \times 10^{-20}$ & $2.150 \times 10^{-10}$ & $1.000 \times 10^{-20}$ & $1.000 \mathrm{E} \times 10^{-20}$ & $2.296 \times 10^{-7}$ & $1.000 \times 10^{-20}$ \\
\hline$I_{\mathrm{o} 3}(\mathrm{~A})$ & $1.0 \times 10^{-20}$ & $5.0 \times 10^{-5}$ & $1.001 \times 10^{-20}$ & $1.325 \times 10^{-9}$ & $2.202 \times 10^{-7}$ & $1.000 \times 10^{-20}$ & $3.270 \times 10^{-20}$ & $8.948 \times 10^{-9}$ \\
\hline$n_{1}$ & 1.0 & 3.0 & 2.623 & 1.096 & 3.000 & 2.635 & 1.029 & 2.997 \\
\hline$n_{2}$ & 1.0 & 5.0 & 1.067 & 2.564 & 1.029 & 1.066 & 5.000 & 1.037 \\
\hline$n_{3}$ & 1.0 & 5.0 & 4.740 & 4.557 & 5.000 & 5.000 & 1.872 & 3.541 \\
\hline$R_{P}$ & 1000 & 7000 & 7000.000 & 7000.000 & 7000.000 & 7000.000 & 7000.000 & 6947.666 \\
\hline$R_{S}$ & 8.000 & 19.00 & 18.000 & 18.173 & 19.000 & 18.000 & 19.000 & 18.842 \\
\hline$R_{s m}$ & 0.001 & 0.30 & 0.191 & 0.203 & 0.300 & 0.296 & 0.142 & 0.103 \\
\hline RMSE & & & $1.379 \times 10^{-5}$ & $1.398 \times 10^{-5}$ & $4.63 \times 10^{-5}$ & $1.38 \times 10^{-5}$ & $4.78 \times 10^{-5}$ & $2.72 \times 10^{-5}$ \\
\hline \multicolumn{9}{|c|}{ Modified Device } \\
\hline$I_{s c}(\mathrm{~A})$ & $1.0 \times 10^{-3}$ & $5.0 \times 10^{-3}$ & $2.450 \times 10^{-3}$ & $2.465 \times 10^{-3}$ & $2.461 \times 10^{-3}$ & $2.452 \times 10^{-3}$ & $2.381 \times 10^{-3}$ & $2.404 \times 10^{-3}$ \\
\hline$I_{\mathrm{o} 1}(\mathrm{~A})$ & $1.0 \times 10^{-20}$ & $5.0 \times 10^{-5}$ & $1.011 \times 10^{-20}$ & $8.699 \times 10^{-11}$ & $1.000 \times 10^{-20}$ & $1.000 \times 10^{-20}$ & $1.000 \times 10^{-20}$ & $1.000 \times 10^{-20}$ \\
\hline$I_{\mathrm{O} 2}(\mathrm{~A})$ & $1.0 \times 10^{-20}$ & $5.0 \times 10^{-5}$ & $1.206 \times 10^{-20}$ & $2.696 \times 10^{-10}$ & $1.998 \times 10^{-9}$ & $3.170 \times 10^{-10}$ & $1.112 \times 10^{-16}$ & $1.150 \times 10^{-8}$ \\
\hline$I_{\mathrm{O} 3}(\mathrm{~A})$ & $1.0 \times 10^{-20}$ & $5.0 \times 10^{-5}$ & $4.874 \times 10^{-11}$ & $1.735 \times 10^{-20}$ & $1.000 \times 10^{-20}$ & $4.838 \times 10^{-13}$ & $1.000 \times 10^{-20}$ & $1.000 \times 10^{-20}$ \\
\hline $\begin{array}{c}{ }_{0} 3(A) \\
n_{1}\end{array}$ & 1.0 & 3.0 & $\begin{array}{r}4.074 \times 10 \\
2.9991\end{array}$ & 2.4717 & 3.0000 & $\begin{array}{c}4.000 \times 10 \\
2.0895\end{array}$ & 1.4302 & 1.8028 \\
\hline$n_{2}$ & 1.0 & 5.0 & 4.9989 & 2.6409 & 2.8916 & 2.6619 & 1.3153 & 3.4279 \\
\hline$n_{3}$ & 1.0 & 5.0 & 2.2890 & 2.0320 & 5.0000 & 1.8821 & 3.8176 & 1.0406 \\
\hline$R_{P}$ & 1000 & 10,000 & $10,000.00$ & 7602.58 & $10,000.00$ & $10,000.00$ & $10,000.00$ & 9514.81 \\
\hline$R_{s}$ & 5.00 & 17.5 & 17.00 & 10.05 & 5.00 & 17.00 & 17.00 & 11.95 \\
\hline$R_{s m}$ & 0.001 & 0.30 & 0.2000 & 0.1876 & 0.2000 & 0.1000 & 0.1571 & 0.1751 \\
\hline RMSE & & & $1.499 \times 10^{-5}$ & $2.269 \times 10^{-5}$ & $2.252 \times 10^{-5}$ & $1.556 \times 10^{-5}$ & $1.188 \times 10^{-4}$ & $6.881 \times 10^{-5}$ \\
\hline
\end{tabular}

Table 3. Statistical performance assessment of considered optimizers using TDM (30 times).

\begin{tabular}{ccccccc}
\hline Metric & BES & GWO & POS & MFO & SCA \\
\hline \multicolumn{7}{c}{ Control Device } \\
\hline Best & $1.379 \times 10^{-5}$ & $1.397 \times 10^{-5}$ & $1.564 \times 10^{-5}$ & $1.379 \times 10^{-5}$ & $3.926 \times 10^{-5}$ & $2.243 \times 10^{-5}$ \\
Worst & $2.019 \times 10^{-5}$ & $1.139 \times 10^{-4}$ & $3.661 \times 10^{-4}$ & $1.134 \times 10^{-4}$ & $1.174 \times 10^{-4}$ & $2.173 \times 10^{-4}$ \\
Average & $1.660 \times 10^{-5}$ & $3.403 \times 10^{-5}$ & $2.039 \times 10^{-4}$ & $3.176 \times 10^{-5}$ & $1.037 \times 10^{-4}$ & $9.393 \times 10^{-5}$ \\
Variance & $1.132 \times 10^{-12}$ & $3.418 \times 10^{-10}$ & $1.173 \times 10^{-8}$ & $6.101 \times 10^{-10}$ & $2.476 \times 10^{-10}$ & $1.713 \times 10^{-9}$ \\
Median & $1.637 \times 10^{-5}$ & $3.236 \times 10^{-5}$ & $1.433 \times 10^{-4}$ & $2.451 \times 10^{-5}$ & $1.083 \times 10^{-4}$ & $8.950 \times 10^{-5}$ \\
STD & $1.064 \times 10^{-6}$ & $1.849 \times 10^{-5}$ & $1.083 \times 10^{-4}$ & $2.470 \times 10^{-5}$ & $1.574 \times 10^{-4}$ & $4.139 \times 10^{-5}$ \\
\hline & & Modified Device & \\
\hline Best & $1.632 \times 10^{-5}$ & $2.033 \times 10^{-5}$ & $3.006 \times 10^{-5}$ & $1.857 \times 10^{-5}$ & $1.013 \times 10^{-4}$ & $5.039 \times 10^{-5}$ \\
Worst & $2.343 \times 10^{-5}$ & $1.527 \times 10^{-4}$ & $1.348 \times 10^{-3}$ & $8.240 \times 10^{-5}$ & $1.902 \times 10^{-4}$ & $1.810 \times 10^{-5}$ \\
Average & $1.689 \times 10^{-5}$ & $5.487 \times 10^{-5}$ & $2.438 \times 10^{-4}$ & $4.117 \times 10^{-5}$ & $1.519 \times 10^{-4}$ & $9.722 \times 10^{-5}$ \\
Variance & $2.622 \times 10^{-12}$ & $8.773 \times 10^{-10}$ & $9.612 \times 10^{-8}$ & $2.817 \times 10^{-10}$ & $3.820 \times 10^{-10}$ & $6.473 \times 10^{-5}$ \\
Median & $1.634 \times 10^{-5}$ & $4.971 \times 10^{-5}$ & $1.591 \times 10^{-4}$ & $3.847 \times 10^{-5}$ & $1.515 \times 10^{-4}$ & $9.330 \times 10^{-5}$ \\
STD & $1.619 \times 10^{-6}$ & $2.962 \times 10^{-5}$ & $3.100 \times 10^{-4}$ & $1.678 \times 10^{-5}$ & $1.954 \times 10^{-5}$ & $2.544 \times 10^{-5}$ \\
\hline
\end{tabular}

Table 4. Statistical performance assessment of considered optimizers using MTDM (30 times).

\begin{tabular}{ccccccc}
\hline Metric & BES & GWO & POS & MFO & SCA & SSA \\
\hline \multicolumn{7}{c}{ Control Device } \\
\hline Best & $1.379 \times 10^{-5}$ & $1.398 \times 10^{-5}$ & $4.629 \times 10^{-5}$ & $1.380 \times 10^{-5}$ & $4.780 \times 10^{-5}$ & $2.716 \times 10^{-5}$ \\
Worst & $2.613 \times 10^{-5}$ & $1.113 \times 10^{-4}$ & $1.182 \times 10^{-3}$ & $4.424 \times 10^{-5}$ & $1.180 \times 10^{-4}$ & $2.095 \times 10^{-4}$ \\
Average & $1.570 \times 10^{-5}$ & $3.154 \times 10^{-5}$ & $2.557 \times 10^{-4}$ & $2.470 \times 10^{-5}$ & $1.021 \times 10^{-4}$ & $8.783 \times 10^{-5}$ \\
Variance & $7.547 \times 10^{-12}$ & $5.790 \times 10^{-10}$ & $7.615 \times 10^{-8}$ & $9.603 \times 10^{-11}$ & $2.517 \times 10^{-10}$ & $1.814 \times 10^{-9}$ \\
Median & $1.514 \times 10^{-5}$ & $2.652 \times 10^{-5}$ & $1.307 \times 10^{-4}$ & $2.237 \times 10^{-5}$ & $1.048 \times 10^{-4}$ & $8.894 \times 10^{-5}$ \\
STD & $2.747 \times 10^{-6}$ & $2.406 \times 10^{-5}$ & $2.760 \times 10^{-4}$ & $9.799 \times 10^{-6}$ & $1.586 \times 10^{-5}$ & $4.259 \times 10^{-5}$ \\
\hline
\end{tabular}


Table 4. Cont.

\begin{tabular}{cccccc}
\hline Metric & BES & GWO & POS & MFO \\
\hline & \multicolumn{3}{c}{ Sodified Device } \\
\hline Best & $1.499 \times 10^{-5}$ & $2.269 \times 10^{-5}$ & $2.252 \times 10^{-5}$ & $1.556 \times 10^{-5}$ & $1.188 \times 10^{-4}$ \\
Worst & $2.252 \times 10^{-5}$ & $1.465 \times 10^{-4}$ & $3.650 \times 10^{-4}$ & $1.363 \times 10^{-4}$ & $1.815 \times 10^{-4}$ \\
Average & $1.536 \times 10^{-5}$ & $7.983 \times 10^{-5}$ & $2.048 \times 10^{-4}$ & $6.024 \times 10^{-5}$ & $1.539 \times 10^{-4}$ \\
Variance & $1.983 \times 10^{-12}$ & $2.060 \times 10^{-9}$ & $9.373 \times 10^{-9}$ & $1.942 \times 10^{-9}$ & $2.251 \times 10^{-10}$ \\
Median & $1.501 \times 10^{-5}$ & $6.754 \times 10^{-5}$ & $1.943 \times 10^{-4}$ & $4.588 \times 10^{-5}$ & $1.215 \times 10^{-4}$ \\
STD & $1.408 \times 10^{-6}$ & $4.539 \times 10^{-5}$ & $9.681 \times 10^{-5}$ & $4.407 \times 10^{-5}$ & $1.500 \times 10^{-5}$ \\
\hline
\end{tabular}

Table 5. Details of 30 TDM Runs for Control Device for each applied optimizer.

\begin{tabular}{|c|c|c|c|c|c|c|}
\hline & BES & GWO & PSO & MFO & SCA & SSA \\
\hline 1 & $1.632 \times 10^{-5}$ & $4.029 \times 10^{-5}$ & $1.169 \times 10^{-4}$ & $1.536 \times 10^{-5}$ & $1.116 \times 10^{-4}$ & $2.173 \times 10^{-4}$ \\
\hline 2 & $1.656 \times 10^{-5}$ & $2.137 \times 10^{-5}$ & $1.167 \times 10^{-4}$ & $1.562 \times 10^{-5}$ & $9.026 \times 10^{-5}$ & $1.154 \times 10^{-4}$ \\
\hline 3 & $1.632 \times 10^{-5}$ & $4.325 \times 10^{-5}$ & $1.306 \times 10^{-4}$ & $1.564 \times 10^{-5}$ & $1.140 \times 10^{-4}$ & $1.304 \times 10^{-4}$ \\
\hline 4 & $1.633 \times 10^{-5}$ & $1.916 \times 10^{-5}$ & $1.451 \times 10^{-4}$ & $1.731 \times 10^{-5}$ & $1.099 \times 10^{-4}$ & $1.185 \times 10^{-4}$ \\
\hline 5 & $1.948 \times 10^{-5}$ & $4.359 \times 10^{-5}$ & $3.661 \times 10^{-4}$ & $3.661 \times 10^{-5}$ & $1.055 \times 10^{-4}$ & $1.028 \times 10^{-4}$ \\
\hline 6 & $1.655 \times 10^{-5}$ & $3.141 \times 10^{-5}$ & $3.196 \times 10^{-4}$ & $3.027 \times 10^{-5}$ & $1.174 \times 10^{-4}$ & $2.638 \times 10^{-5}$ \\
\hline 7 & $2.019 \times 10^{-5}$ & $2.622 \times 10^{-5}$ & $1.169 \times 10^{-4}$ & $2.266 \times 10^{-5}$ & $8.740 \times 10^{-5}$ & $8.144 \times 10^{-5}$ \\
\hline 8 & $1.632 \times 10^{-5}$ & $1.397 \times 10^{-5}$ & $3.199 \times 10^{-4}$ & $1.548 \times 10^{-5}$ & $1.160 \times 10^{-4}$ & $1.209 \times 10^{-4}$ \\
\hline 9 & $1.632 \times 10^{-5}$ & $3.913 \times 10^{-5}$ & $3.196 \times 10^{-4}$ & $5.485 \times 10^{-5}$ & $1.110 \times 10^{-4}$ & $2.243 \times 10^{-5}$ \\
\hline 10 & $1.632 \times 10^{-5}$ & $3.331 \times 10^{-5}$ & $1.564 \times 10^{-5}$ & $3.041 \times 10^{-5}$ & $1.045 \times 10^{-4}$ & $9.011 \times 10^{-4}$ \\
\hline 11 & $1.653 \times 10^{-5}$ & $2.579 \times 10^{-5}$ & $1.169 \times 10^{-4}$ & $3.259 \times 10^{-5}$ & $1.123 \times 10^{-4}$ & $6.787 \times 10^{-4}$ \\
\hline 12 & $1.639 \times 10^{-5}$ & $3.566 \times 10^{-5}$ & $3.199 \times 10^{-4}$ & $1.134 \times 10^{-4}$ & $1.074 \times 10^{-4}$ & $1.217 \times 10^{-4}$ \\
\hline 13 & $1.632 \times 10^{-5}$ & $4.502 \times 10^{-5}$ & $1.169 \times 10^{-4}$ & $3.021 \times 10^{-5}$ & $1.124 \times 10^{-4}$ & $1.134 \times 10^{-4}$ \\
\hline 14 & $1.638 \times 10^{-5}$ & $4.032 \times 10^{-5}$ & $3.199 \times 10^{-4}$ & $2.841 \times 10^{-5}$ & $7.686 \times 10^{-5}$ & $1.392 \times 10^{-4}$ \\
\hline 15 & $1.379 \times 10^{-5}$ & $2.157 \times 10^{-5}$ & $1.451 \times 10^{-4}$ & $4.141 \times 10^{-5}$ & $3.926 \times 10^{-5}$ & $4.130 \times 10^{-5}$ \\
\hline 16 & $1.650 \times 10^{-5}$ & $2.163 \times 10^{-5}$ & $1.323 \times 10^{-4}$ & $2.098 \times 10^{-5}$ & $1.027 \times 10^{-4}$ & $8.517 \times 10^{-5}$ \\
\hline 17 & $1.637 \times 10^{-5}$ & $4.492 \times 10^{-5}$ & $3.196 \times 10^{-4}$ & $1.690 \times 10^{-5}$ & $1.166 \times 10^{-4}$ & $1.098 \times 10^{-4}$ \\
\hline 18 & $1.639 \times 10^{-5}$ & $2.398 \times 10^{-5}$ & $3.199 \times 10^{-4}$ & $1.432 \times 10^{-5}$ & $1.092 \times 10^{-4}$ & $1.244 \times 10^{-4}$ \\
\hline 19 & $1.632 \times 10^{-5}$ & $4.417 \times 10^{-5}$ & $3.199 \times 10^{-4}$ & $4.632 \times 10^{-5}$ & $8.308 \times 10^{-5}$ & $1.490 \times 10^{-4}$ \\
\hline 20 & $1.641 \times 10^{-5}$ & $1.640 \times 10^{-5}$ & $1.195 \times 10^{-4}$ & $1.134 \times 10^{-4}$ & $1.051 \times 10^{-4}$ & $4.998 \times 10^{-5}$ \\
\hline 21 & $1.672 \times 10^{-5}$ & $1.922 \times 10^{-5}$ & $1.195 \times 10^{-4}$ & $1.379 \times 10^{-5}$ & $1.133 \times 10^{-4}$ & $4.712 \times 10^{-5}$ \\
\hline 22 & $1.632 \times 10^{-5}$ & $1.762 \times 10^{-5}$ & $3.196 \times 10^{-4}$ & $1.887 \times 10^{-5}$ & $1.111 \times 10^{-4}$ & $7.103 \times 10^{-5}$ \\
\hline 23 & $1.645 \times 10^{-5}$ & $2.464 \times 10^{-5}$ & $1.822 \times 10^{-5}$ & $4.452 \times 10^{-5}$ & $1.020 \times 10^{-4}$ & $8.050 \times 10^{-5}$ \\
\hline 24 & $1.637 \times 10^{-5}$ & $4.481 \times 10^{-5}$ & $1.306 \times 10^{-4}$ & $2.886 \times 10^{-5}$ & $1.063 \times 10^{-4}$ & $4.989 \times 10^{-5}$ \\
\hline 25 & $1.636 \times 10^{-5}$ & $3.511 \times 10^{-5}$ & $1.323 \times 10^{-4}$ & $2.636 \times 10^{-5}$ & $9.886 \times 10^{-5}$ & $8.166 \times 10^{-5}$ \\
\hline 26 & $1.632 \times 10^{-5}$ & $2.663 \times 10^{-5}$ & $3.196 \times 10^{-4}$ & $3.610 \times 10^{-5}$ & $1.037 \times 10^{-4}$ & $8.889 \times 10^{-5}$ \\
\hline 27 & $1.817 \times 10^{-5}$ & $4.520 \times 10^{-5}$ & $2.886 \times 10^{-4}$ & $1.957 \times 10^{-5}$ & $1.039 \times 10^{-4}$ & $1.091 \times 10^{-4}$ \\
\hline 28 & $1.642 \times 10^{-5}$ & $4.501 \times 10^{-5}$ & $3.196 \times 10^{-4}$ & $1.611 \times 10^{-5}$ & $1.094 \times 10^{-4}$ & $4.720 \times 10^{-5}$ \\
\hline 29 & $1.632 \times 10^{-5}$ & $1.764 \times 10^{-5}$ & $1.416 \times 10^{-4}$ & $1.424 \times 10^{-5}$ & $1.160 \times 10^{-4}$ & $1.270 \times 10^{-4}$ \\
\hline 30 & $1.632 \times 10^{-5}$ & $1.139 \times 10^{-4}$ & $1.323 \times 10^{-4}$ & $2.231 \times 10^{-5}$ & $1.140 \times 10^{-4}$ & $8.815 \times 10^{-5}$ \\
\hline
\end{tabular}

Table 6. Details of 30 TDM Runs for Modified Device for each applied optimizer.

\begin{tabular}{ccccccc}
\hline & BES & GWO & PSO & MFO & SCA \\
\hline 1 & $1.64 \times 10^{-5}$ & $6.28 \times 10^{-5}$ & 0.0001257 & $2.49 \times 10^{-5}$ & 0.0001779 \\
2 & $1.63 \times 10^{-5}$ & $6.33 \times 10^{-5}$ & $6.19 \times 10^{-5}$ & $7.29 \times 10^{-5}$ & 0.000134006 & $0.18 \times 10^{-5}$ \\
3 & $1.65 \times 10^{-5}$ & $3.14 \times 10^{-5}$ & 0.000163679 & $8.24 \times 10^{-5}$ & 0.000142692 & 0.000108355 \\
4 & $1.63 \times 10^{-5}$ & $2.39 \times 10^{-5}$ & $3.01 \times 10^{-5}$ & $1.86 \times 10^{-5}$ & 0.000151738 & 0.000102329 \\
5 & $1.63 \times 10^{-5}$ & $1.36 \times 10^{-5}$ & 0.000281539 & $6.12 \times 10^{-5}$ & 0.000158538 & $7.87 \times 10^{-5}$ \\
6 & $1.63 \times 10^{-5}$ & $7.03 \times 10^{-5}$ & 0.000295073 & $3.85 \times 10^{-5}$ & 0.000173369 & $8.26 \times 10^{-5}$ \\
7 & $1.68 \times 10^{-5}$ & $6.08 \times 10^{-5}$ & 0.0001257 & $2.25 \times 10^{-5}$ & 0.000165133 \\
8 & $1.64 \times 10^{-5}$ & $2.95 \times 10^{-5}$ & 0.0001257 & $5.35 \times 10^{-5}$ & 0.000151567 & $7.99 \times 10^{-5}$ \\
\hline
\end{tabular}


Table 6. Cont.

\begin{tabular}{|c|c|c|c|c|c|c|}
\hline & BES & GWO & PSO & MFO & SCA & SSA \\
\hline 9 & $1.63 \times 10^{-5}$ & $4.75 \times 10^{-5}$ & 0.001348167 & $3.07 \times 10^{-5}$ & 0.000148697 & $9.28 \times 10^{-5}$ \\
\hline 10 & $1.64 \times 10^{-5}$ & $8.32 \times 10^{-5}$ & 0.000205182 & $6.06 \times 10^{-5}$ & 0.000175416 & 0.000121079 \\
\hline 11 & $1.63 \times 10^{-5}$ & $3.20 \times 10^{-5}$ & 0.000211831 & $5.26 \times 10^{-5}$ & 0.00013459 & $5.07 \times 10^{-5}$ \\
\hline 12 & $1.63 \times 10^{-5}$ & $4.23 \times 10^{-5}$ & 0.000154443 & $1.90 \times 10^{-5}$ & 0.000172478 & $9.90 \times 10^{-5}$ \\
\hline 13 & $1.64 \times 10^{-5}$ & $6.47 \times 10^{-5}$ & 0.000205182 & $3.98 \times 10^{-5}$ & 0.00014998 & $9.92 \times 10^{-5}$ \\
\hline 14 & $1.65 \times 10^{-5}$ & $3.46 \times 10^{-5}$ & $6.19 \times 10^{-5}$ & $4.43 \times 10^{-5}$ & 0.000147608 & 0.000133438 \\
\hline 15 & $1.63 \times 10^{-5}$ & $2.11 \times 10^{-5}$ & 0.000154443 & $5.34 \times 10^{-5}$ & 0.000146388 & $9.21 \times 10^{-5}$ \\
\hline 16 & $1.68 \times 10^{-5}$ & $3.59 \times 10^{-5}$ & 0.000163679 & $6.02 \times 10^{-5}$ & 0.000101269 & 0.000180993 \\
\hline 17 & $1.63 \times 10^{-5}$ & $7.17 \times 10^{-5}$ & 0.0001257 & $4.26 \times 10^{-5}$ & 0.000161644 & $8.93 \times 10^{-5}$ \\
\hline 18 & $1.63 \times 10^{-5}$ & $4.81 \times 10^{-5}$ & 0.000163679 & $3.10 \times 10^{-5}$ & 0.000151409 & $5.04 \times 10^{-5}$ \\
\hline 19 & $2.15 \times 10^{-5}$ & $5.53 \times 10^{-5}$ & 0.0001257 & $5.46 \times 10^{-5}$ & 0.000153947 & 0.00010374 \\
\hline 20 & $1.63 \times 10^{-5}$ & $5.32 \times 10^{-5}$ & 0.000360627 & $2.09 \times 10^{-5}$ & 0.000148987 & $8.89 \times 10^{-5}$ \\
\hline 21 & $1.64 \times 10^{-5}$ & $4.75 \times 10^{-5}$ & 0.0001257 & $3.44 \times 10^{-5}$ & 0.000137175 & $9.38 \times 10^{-5}$ \\
\hline 22 & $1.63 \times 10^{-5}$ & $4.02 \times 10^{-5}$ & $6.19 \times 10^{-5}$ & $2.22 \times 10^{-5}$ & 0.000153647 & 0.000136316 \\
\hline 23 & $1.63 \times 10^{-5}$ & 0.000152694 & 0.000211831 & $3.70 \times 10^{-5}$ & 0.000141687 & $9.91 \times 10^{-5}$ \\
\hline 24 & $1.63 \times 10^{-5}$ & $6.38 \times 10^{-5}$ & 0.0001257 & $3.25 \times 10^{-5}$ & 0.0001776 & $6.85 \times 10^{-5}$ \\
\hline 25 & $1.65 \times 10^{-5}$ & $2.03 \times 10^{-5}$ & 0.000281539 & $2.65 \times 10^{-5}$ & 0.000190216 & $8.30 \times 10^{-5}$ \\
\hline 26 & $1.92 \times 10^{-5}$ & $5.14 \times 10^{-5}$ & $6.19 \times 10^{-5}$ & $3.85 \times 10^{-5}$ & 0.000155325 & 0.00010078 \\
\hline 27 & $1.63 \times 10^{-5}$ & $6.19 \times 10^{-5}$ & 0.0001257 & $2.60 \times 10^{-5}$ & 0.0001694 & $9.03 \times 10^{-5}$ \\
\hline 28 & $1.63 \times 10^{-5}$ & $2.59 \times 10^{-5}$ & 0.000203116 & $3.02 \times 10^{-5}$ & 0.000132844 & $7.40 \times 10^{-5}$ \\
\hline 29 & $2.34 \times 10^{-5}$ & $6.97 \times 10^{-5}$ & 0.000281539 & $6.05 \times 10^{-5}$ & 0.000146441 & 0.000111004 \\
\hline 30 & $1.65 \times 10^{-5}$ & $4.48 \times 10^{-5}$ & 0.001346458 & $4.31 \times 10^{-5}$ & 0.000105617 & 0.000113337 \\
\hline
\end{tabular}

Table 7. Details of 30 MTDM Runs for Control Device for each applied optimizer.

\begin{tabular}{|c|c|c|c|c|c|c|}
\hline & BES & GWO & PSO & MFO & SCA & SSA \\
\hline 1 & $1.438 \times 10^{-5}$ & $1.586 \times 10^{-5}$ & $3.196 \times 10^{-4}$ & $3.582 \times 10^{-5}$ & $1.023 \times 10^{-4}$ & $3.227 \times 10^{-4}$ \\
\hline 2 & $1.380 \times 10^{-5}$ & $3.096 \times 10^{-5}$ & $4.486 \times 10^{-4}$ & $2.938 \times 10^{-5}$ & $1.005 \times 10^{-4}$ & $3.681 \times 10^{-4}$ \\
\hline 3 & $1.525 \times 10^{-5}$ & $1.570 \times 10^{-5}$ & $3.196 \times 10^{-4}$ & $1.787 \times 10^{-5}$ & $1.147 \times 10^{-4}$ & $3.347 \times 10^{-4}$ \\
\hline 4 & $1.525 \times 10^{-5}$ & $1.486 \times 10^{-5}$ & $1.140 \times 10^{-4}$ & $1.405 \times 10^{-5}$ & $9.289 \times 10^{-5}$ & $4.556 \times 10^{-4}$ \\
\hline 5 & $1.560 \times 10^{-5}$ & $2.476 \times 10^{-5}$ & $3.661 \times 10^{-4}$ & $3.484 \times 10^{-5}$ & $1.041 \times 10^{-4}$ & $3.114 \times 10^{-4}$ \\
\hline 6 & $1.564 \times 10^{-5}$ & $1.459 \times 10^{-5}$ & $1.182 \times 10^{-3}$ & $4.424 \times 10^{-5}$ & $7.503 \times 10^{-5}$ & $1.533 \times 10^{-4}$ \\
\hline 7 & $1.379 \times 10^{-5}$ & $1.563 \times 10^{-5}$ & $1.169 \times 10^{-4}$ & $1.458 \times 10^{-5}$ & $1.161 \times 10^{-4}$ & $4.864 \times 10^{-4}$ \\
\hline 8 & $2.441 \times 10^{-5}$ & $4.392 \times 10^{-5}$ & $1.306 \times 10^{-4}$ & $3.978 \times 10^{-5}$ & $1.054 \times 10^{-4}$ & $5.956 \times 10^{-4}$ \\
\hline 9 & $1.380 \times 10^{-5}$ & $3.551 \times 10^{-5}$ & $1.183 \times 10^{-4}$ & $2.737 \times 10^{-5}$ & $1.142 \times 10^{-4}$ & $1.534 \times 10^{-4}$ \\
\hline 10 & $1.496 \times 10^{-5}$ & $3.336 \times 10^{-5}$ & $1.181 \times 10^{-3}$ & $4.289 \times 10^{-5}$ & $8.310 \times 10^{-5}$ & $1.020 \times 10^{-3}$ \\
\hline 11 & $1.460 \times 10^{-5}$ & $3.079 \times 10^{-5}$ & $1.306 \times 10^{-4}$ & $1.380 \times 10^{-5}$ & $1.180 \times 10^{-4}$ & $1.636 \times 10^{-4}$ \\
\hline 12 & $2.613 \times 10^{-5}$ & $4.400 \times 10^{-5}$ & $1.183 \times 10^{-4}$ & $2.321 \times 10^{-5}$ & $1.009 \times 10^{-4}$ & $3.891 \times 10^{-4}$ \\
\hline 13 & $1.552 \times 10^{-5}$ & $1.098 \times 10^{-4}$ & $3.196 \times 10^{-4}$ & $1.540 \times 10^{-5}$ & $1.104 \times 10^{-4}$ & $1.403 \times 10^{-4}$ \\
\hline 14 & $1.535 \times 10^{-5}$ & $2.828 \times 10^{-5}$ & $3.196 \times 10^{-4}$ & $1.527 \times 10^{-5}$ & $9.489 \times 10^{-5}$ & $2.843 \times 10^{-4}$ \\
\hline 15 & $1.468 \times 10^{-5}$ & $1.821 \times 10^{-5}$ & $1.156 \times 10^{-4}$ & $1.877 \times 10^{-5}$ & $1.024 \times 10^{-4}$ & $8.228 \times 10^{-4}$ \\
\hline 16 & $1.443 \times 10^{-5}$ & $4.393 \times 10^{-5}$ & $3.661 \times 10^{-4}$ & $2.153 \times 10^{-5}$ & $1.141 \times 10^{-4}$ & $2.957 \times 10^{-4}$ \\
\hline 17 & $1.498 \times 10^{-5}$ & $1.765 \times 10^{-5}$ & $1.306 \times 10^{-4}$ & $2.031 \times 10^{-5}$ & $1.028 \times 10^{-4}$ & $1.676 \times 10^{-4}$ \\
\hline 18 & $1.772 \times 10^{-5}$ & $2.890 \times 10^{-5}$ & $1.308 \times 10^{-4}$ & $2.650 \times 10^{-5}$ & $1.157 \times 10^{-4}$ & $6.229 \times 10^{-4}$ \\
\hline 19 & $1.522 \times 10^{-5}$ & $2.211 \times 10^{-5}$ & $1.380 \times 10^{-4}$ & $2.657 \times 10^{-5}$ & $4.780 \times 10^{-5}$ & $1.941 \times 10^{-4}$ \\
\hline 20 & $1.497 \times 10^{-5}$ & $1.562 \times 10^{-5}$ & $4.629 \times 10^{-5}$ & $4.122 \times 10^{-5}$ & $1.098 \times 10^{-4}$ & $4.673 \times 10^{-4}$ \\
\hline 21 & $1.522 \times 10^{-5}$ & $1.398 \times 10^{-5}$ & $1.169 \times 10^{-4}$ & $1.658 \times 10^{-5}$ & $1.171 \times 10^{-4}$ & $4.503 \times 10^{-4}$ \\
\hline 22 & $1.583 \times 10^{-5}$ & $1.113 \times 10^{-4}$ & $3.196 \times 10^{-4}$ & $2.497 \times 10^{-5}$ & $1.020 \times 10^{-4}$ & $2.981 \times 10^{-4}$ \\
\hline 23 & $1.380 \times 10^{-5}$ & $3.813 \times 10^{-5}$ & $1.416 \times 10^{-4}$ & $1.916 \times 10^{-5}$ & $1.115 \times 10^{-4}$ & $3.998 \times 10^{-4}$ \\
\hline 24 & $1.506 \times 10^{-5}$ & $1.596 \times 10^{-5}$ & $4.629 \times 10^{-5}$ & $3.023 \times 10^{-5}$ & $8.138 \times 10^{-5}$ & $1.381 \times 10^{-3}$ \\
\hline 25 & $1.432 \times 10^{-5}$ & $4.303 \times 10^{-5}$ & $1.306 \times 10^{-4}$ & $1.427 \times 10^{-5}$ & $9.467 \times 10^{-5}$ & $1.613 \times 10^{-4}$ \\
\hline 26 & $1.509 \times 10^{-5}$ & $1.481 \times 10^{-5}$ & $1.145 \times 10^{-4}$ & $1.555 \times 10^{-5}$ & $7.807 \times 10^{-5}$ & $3.663 \times 10^{-4}$ \\
\hline 27 & $1.663 \times 10^{-5}$ & $1.533 \times 10^{-5}$ & $3.661 \times 10^{-4}$ & $2.766 \times 10^{-5}$ & $1.107 \times 10^{-4}$ & $3.586 \times 10^{-4}$ \\
\hline 28 & $1.379 \times 10^{-5}$ & $4.520 \times 10^{-5}$ & $1.306 \times 10^{-4}$ & $1.524 \times 10^{-5}$ & $1.175 \times 10^{-4}$ & $7.003 \times 10^{-4}$ \\
\hline 29 & $1.555 \times 10^{-5}$ & $2.832 \times 10^{-5}$ & $4.716 \times 10^{-5}$ & $1.566 \times 10^{-5}$ & $1.115 \times 10^{-4}$ & $3.501 \times 10^{-4}$ \\
\hline 30 & $1.519 \times 10^{-5}$ & $1.560 \times 10^{-5}$ & $1.451 \times 10^{-4}$ & $3.837 \times 10^{-5}$ & $1.129 \times 10^{-4}$ & $1.604 \times 10^{-4}$ \\
\hline
\end{tabular}


Table 8. Details of 30 MTDM Runs for Modified Device for each applied optimizer.

\begin{tabular}{|c|c|c|c|c|c|c|}
\hline & BES & GWO & PSO & MFO & SCA & SSA \\
\hline 1 & $1.503 \times 10^{-5}$ & $2.782 \times 10^{-5}$ & $3.607 \times 10^{-5}$ & $2.655 \times 10^{-5}$ & $1.551 \times 10^{-4}$ & $1.410 \times 10^{-4}$ \\
\hline 2 & $1.501 \times 10^{-5}$ & $5.764 \times 10^{-5}$ & $2.277 \times 10^{-5}$ & $2.898 \times 10^{-5}$ & $1.296 \times 10^{-4}$ & $7.170 \times 10^{-5}$ \\
\hline 3 & $1.501 \times 10^{-5}$ & $7.363 \times 10^{-5}$ & $7.121 \times 10^{-5}$ & $2.601 \times 10^{-5}$ & $1.590 \times 10^{-4}$ & $1.293 \times 10^{-4}$ \\
\hline 4 & $1.501 \times 10^{-5}$ & $1.352 \times 10^{-4}$ & $1.772 \times 10^{-4}$ & $5.617 \times 10^{-5}$ & $1.701 \times 10^{-4}$ & $8.463 \times 10^{-5}$ \\
\hline 5 & $1.501 \times 10^{-5}$ & $6.892 \times 10^{-5}$ & $2.031 \times 10^{-4}$ & $5.766 \times 10^{-5}$ & $1.589 \times 10^{-4}$ & $1.238 \times 10^{-4}$ \\
\hline 6 & $1.503 \times 10^{-5}$ & $2.276 \times 10^{-5}$ & $1.532 \times 10^{-4}$ & $1.336 \times 10^{-4}$ & $1.603 \times 10^{-4}$ & $1.401 \times 10^{-4}$ \\
\hline 7 & $1.507 \times 10^{-5}$ & $1.350 \times 10^{-4}$ & $3.650 \times 10^{-4}$ & $6.008 \times 10^{-5}$ & $1.687 \times 10^{-4}$ & $1.546 \times 10^{-4}$ \\
\hline 8 & $2.252 \times 10^{-5}$ & $6.277 \times 10^{-5}$ & $7.142 \times 10^{-5}$ & $3.435 \times 10^{-5}$ & $1.633 \times 10^{-4}$ & $9.096 \times 10^{-5}$ \\
\hline 9 & $1.501 \times 10^{-5}$ & $6.969 \times 10^{-5}$ & $1.382 \times 10^{-4}$ & $1.363 \times 10^{-4}$ & $1.462 \times 10^{-4}$ & $7.845 \times 10^{-5}$ \\
\hline 10 & $1.501 \times 10^{-5}$ & $1.344 \times 10^{-4}$ & $2.031 \times 10^{-4}$ & $5.617 \times 10^{-5}$ & $1.389 \times 10^{-4}$ & $8.412 \times 10^{-5}$ \\
\hline 11 & $1.529 \times 10^{-5}$ & $7.032 \times 10^{-5}$ & $3.607 \times 10^{-4}$ & $1.786 \times 10^{-5}$ & $1.725 \times 10^{-4}$ & $1.623 \times 10^{-4}$ \\
\hline 12 & $1.501 \times 10^{-5}$ & $4.648 \times 10^{-5}$ & $1.943 \times 10^{-4}$ & $4.600 \times 10^{-5}$ & $1.588 \times 10^{-4}$ & $1.551 \times 10^{-4}$ \\
\hline 13 & $1.501 \times 10^{-5}$ & $6.711 \times 10^{-5}$ & $1.784 \times 10^{-4}$ & $1.346 \times 10^{-4}$ & $1.544 \times 10^{-4}$ & $1.056 \times 10^{-4}$ \\
\hline 14 & $1.503 \times 10^{-5}$ & $1.465 \times 10^{-4}$ & $1.653 \times 10^{-4}$ & $3.032 \times 10^{-5}$ & $1.734 \times 10^{-4}$ & $7.163 \times 10^{-5}$ \\
\hline 15 & $1.501 \times 10^{-5}$ & $1.410 \times 10^{-4}$ & $1.241 \times 10^{-4}$ & $1.339 \times 10^{-4}$ & $1.293 \times 10^{-4}$ & $1.499 \times 10^{-4}$ \\
\hline 16 & $1.514 \times 10^{-5}$ & $4.345 \times 10^{-5}$ & $1.242 \times 10^{-4}$ & $4.442 \times 10^{-5}$ & $1.188 \times 10^{-4}$ & $1.856 \times 10^{-4}$ \\
\hline 17 & $1.500 \times 10^{-5}$ & $4.363 \times 10^{-5}$ & $3.607 \times 10^{-4}$ & $1.556 \times 10^{-5}$ & $1.471 \times 10^{-4}$ & $1.323 \times 10^{-4}$ \\
\hline 18 & $1.501 \times 10^{-5}$ & $5.769 \times 10^{-5}$ & $2.814 \times 10^{-4}$ & $3.100 \times 10^{-5}$ & $1.481 \times 10^{-4}$ & $9.320 \times 10^{-5}$ \\
\hline 19 & $1.520 \times 10^{-5}$ & $1.360 \times 10^{-4}$ & $1.241 \times 10^{-4}$ & $6.666 \times 10^{-5}$ & $1.544 \times 10^{-4}$ & $1.256 \times 10^{-4}$ \\
\hline 20 & $1.499 \times 10^{-5}$ & $6.797 \times 10^{-5}$ & $2.179 \times 10^{-4}$ & $1.562 \times 10^{-5}$ & $1.260 \times 10^{-4}$ & $7.930 \times 10^{-5}$ \\
\hline 21 & $1.501 \times 10^{-5}$ & $2.969 \times 10^{-5}$ & $1.943 \times 10^{-4}$ & $1.355 \times 10^{-4}$ & $1.815 \times 10^{-4}$ & $1.458 \times 10^{-4}$ \\
\hline 22 & $1.499 \times 10^{-5}$ & $1.369 \times 10^{-4}$ & $2.043 \times 10^{-4}$ & $2.467 \times 10^{-5}$ & $1.411 \times 10^{-4}$ & $1.117 \times 10^{-4}$ \\
\hline 23 & $1.501 \times 10^{-5}$ & $1.454 \times 10^{-4}$ & $2.043 \times 10^{-4}$ & $4.577 \times 10^{-5}$ & $1.683 \times 10^{-4}$ & $1.074 \times 10^{-4}$ \\
\hline 24 & $1.506 \times 10^{-5}$ & $4.847 \times 10^{-5}$ & $1.369 \times 10^{-4}$ & $2.964 \times 10^{-5}$ & $1.617 \times 10^{-4}$ & $6.881 \times 10^{-5}$ \\
\hline 25 & $1.501 \times 10^{-5}$ & $3.715 \times 10^{-5}$ & $3.606 \times 10^{-4}$ & $5.041 \times 10^{-5}$ & $1.441 \times 10^{-4}$ & $1.846 \times 10^{-4}$ \\
\hline 26 & $1.716 \times 10^{-5}$ & $5.522 \times 10^{-5}$ & $2.814 \times 10^{-4}$ & $2.684 \times 10^{-5}$ & $1.512 \times 10^{-4}$ & $9.823 \times 10^{-5}$ \\
\hline 27 & $1.501 \times 10^{-5}$ & $1.455 \times 10^{-4}$ & $3.607 \times 10^{-4}$ & $1.348 \times 10^{-4}$ & $1.539 \times 10^{-4}$ & $8.604 \times 10^{-5}$ \\
\hline 28 & $1.507 \times 10^{-5}$ & $2.549 \times 10^{-5}$ & $1.382 \times 10^{-4}$ & $1.360 \times 10^{-4}$ & $1.580 \times 10^{-4}$ & $7.030 \times 10^{-5}$ \\
\hline 29 & $1.501 \times 10^{-5}$ & $1.405 \times 10^{-4}$ & $2.252 \times 10^{-5}$ & $2.129 \times 10^{-5}$ & $1.689 \times 10^{-4}$ & $9.680 \times 10^{-5}$ \\
\hline 30 & $1.499 \times 10^{-5}$ & $2.269 \times 10^{-5}$ & $1.382 \times 10^{-4}$ & $5.066 \times 10^{-5}$ & $1.550 \times 10^{-4}$ & $1.432 \times 10^{-4}$ \\
\hline
\end{tabular}

From both Tables 3 and 4, the superiority of the proposed BES algorithm is evident for all studied cases. It is clear that the proposed BES algorithm has the best performance compared with other optimizers used in this work. From Table 3, for the control device under the case of TDM, the mean RMSE values have fluctuated between $1.660 \times 10^{-5}$ and $2.039 \times 10^{-4}$. The minimum mean RMSE of $1.660 \times 10^{-5}$ is accomplished by the BES algorithm flowed by $3.176 \times 10^{-5}$ applying the MFO algorithm. The minimum cost function of $1.379 \times 10^{-5}$ is obtained by the BES algorithm followed by MFO and GWO algorithms, respectively. On the other hand, the mean RMSE values for the modified device have fluctuated between $1.689 \times 10^{-5}$ and $2.438 \times 10^{-4}$. The minimum mean RMSE of $1.689 \times 10^{-5}$ is accomplished by the BES algorithm flowed by $4.117 \times 10^{-5}$ applying the MFO algorithm. The minimum cost function of $1.632 \times 10^{-5}$ was obtained by the BES algorithm followed by the MFO algorithm. From Table 4, for the control device under the case of MTDM, the mean RMSE values have fluctuated between $1.570 \times 10^{-5}$ and $2.557 \times 10^{-4}$. The minimum mean RMSE of $1.570 \times 10^{-5}$ is accomplished by the BES algorithm flowed by $2.470 \times 10^{-5}$ applying the MFO algorithm. The minimum cost function of $1.379 \times 10^{-5}$ is obtained by the BES algorithm followed by MFO and GWO algorithms, respectively. In the other side, for the modified device, the mean RMSE values have fluctuated between $1.536 \times 10^{-5}$ and $2.048 \times 10^{-4}$. The minimum mean RMSE of $1.536 \times 10^{-5}$ is accomplished by the BES algorithm flowed by $6.024 \times 10^{-5}$ applying the MFO algorithm. The minimum cost function of $1.499 \times 10^{-5}$ is obtained by the BES algorithm followed by the MFO algorithm. 
The comparison between experimentally obtained and estimated I-V and P-V curves for both control and modified devices used in this study are shown in Figures 8-10. The comparison revealed that the experimental obtained and estimated I-V and $\mathrm{P}-\mathrm{V}$ curves are almost identical, which is an indication of the efficiency of both the suggested model and the optimization algorithm in simulating the performance of the PSCs. The estimated parameters of the PSCs are matched very well with the experimental ones. From previous discussion for Tables 1-4, applying BES algorithm in this study for PSCs parameters estimation via both TDM and MTDM provides the best performance results at minimum errors.

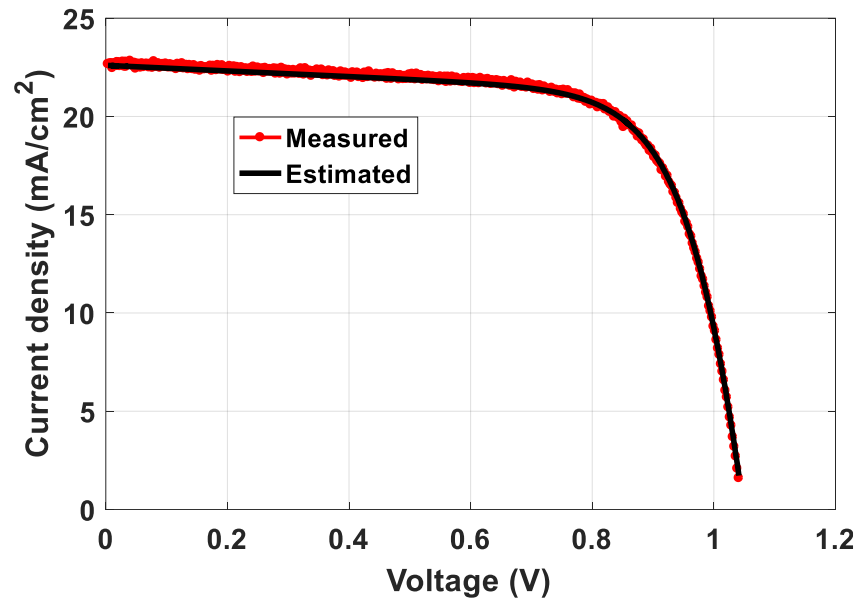

(a)

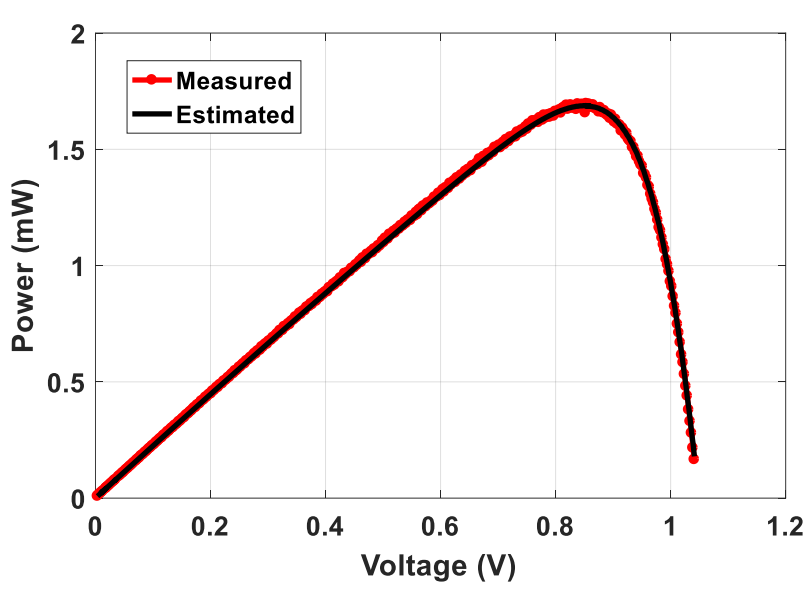

(b)

Figure 8. Output characteristic of control device using BES optimizer (TDM). (a) I-V characteristic of control device; (b) $\mathrm{P}-\mathrm{V}$ characteristic of control device.

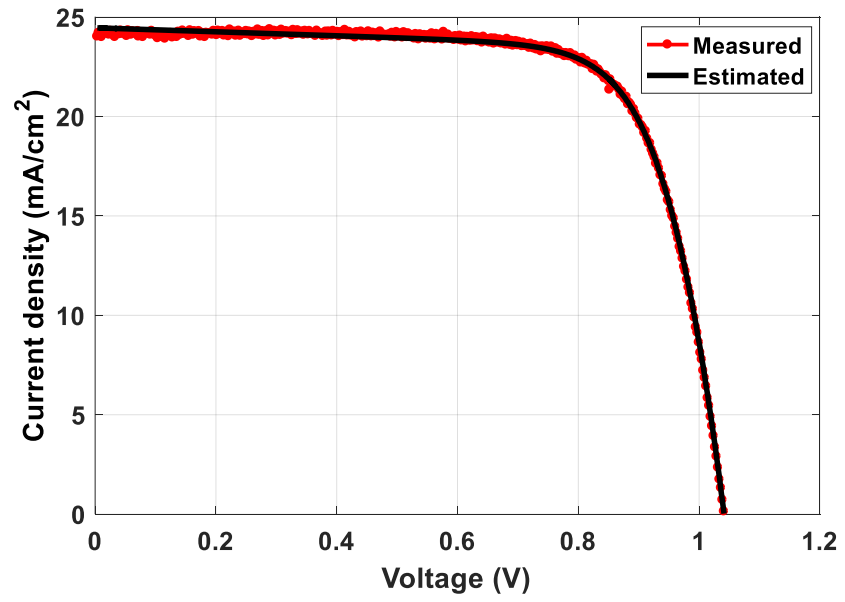

(a)

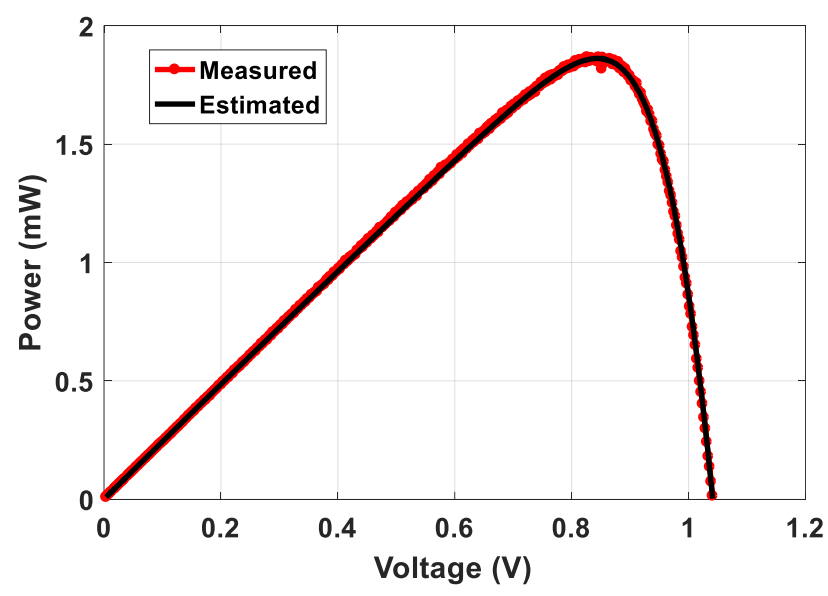

(b)

Figure 9. Output characteristic of modified device using BES optimizer (TDM). (a) I-V characteristic of modified device; (b) P-V characteristic of modified device.

The convergence curves through the parameter identification process of both control and modified devices based on both TDM and MTDM per applying different optimizers are summarized, respectively, in Figures 11 and 12. From these figures, it is obvious that the proposed BES algorithm performance is the best among the other used algorithms. The fast convergence and the lowest cost function resulted from the BES algorithm in all studied cases, which is proof also of the superiority of the BES in comparison with the other optimizers used in this study. 

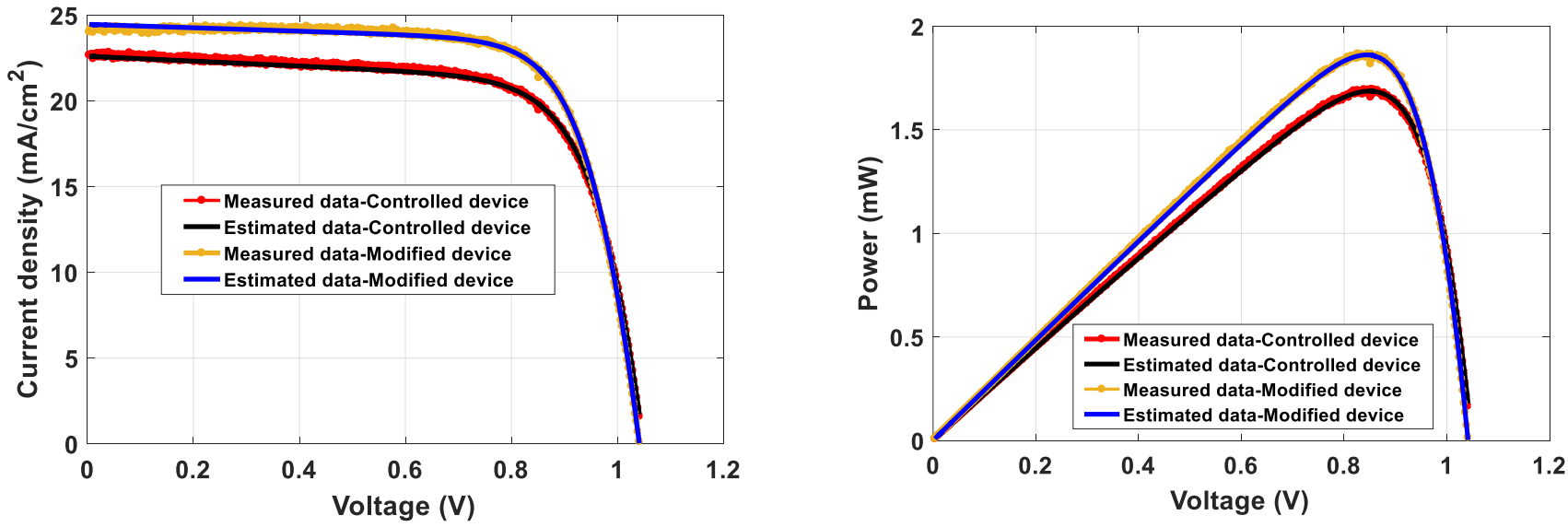

Figure 10. Output characteristic of both modified and controlled devices using BES optimizer (MTDM).

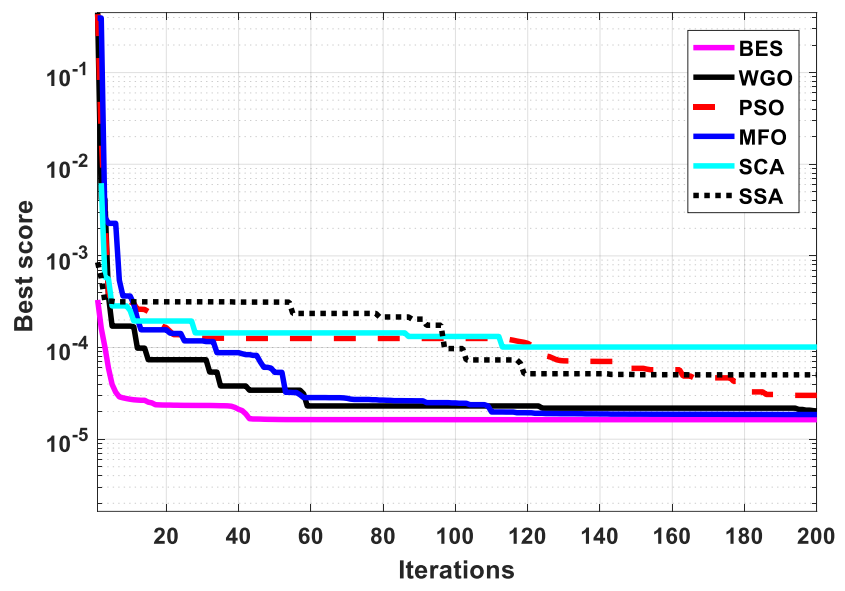

(a)

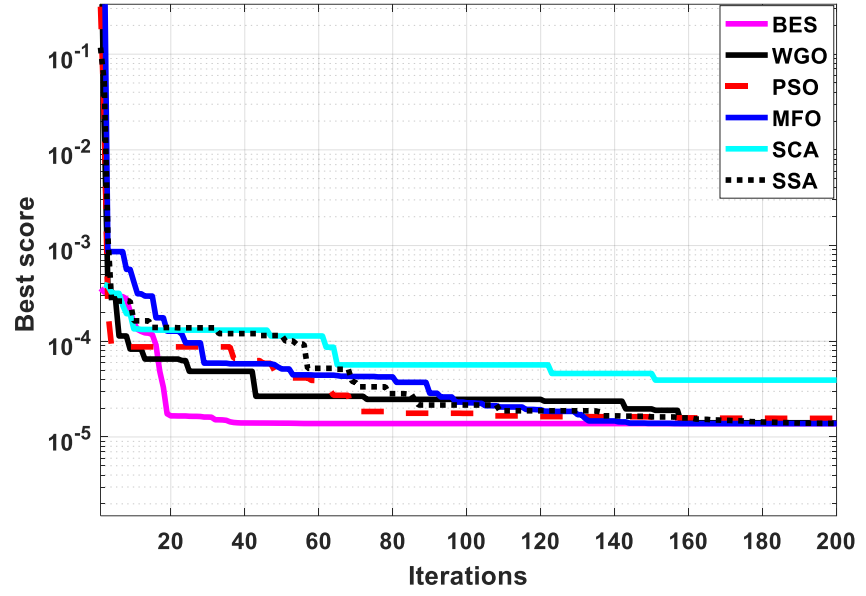

(b)

Figure 11. Convergence curves of parameter estimation using different optimizers. (a) Convergence curve of controlled device (TDM) (b) Convergence curve of modified device (TDM).

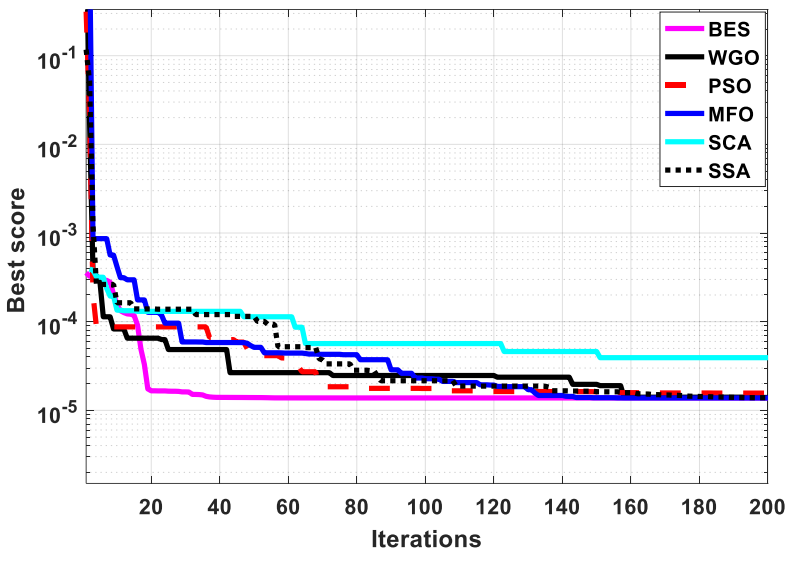

(a)

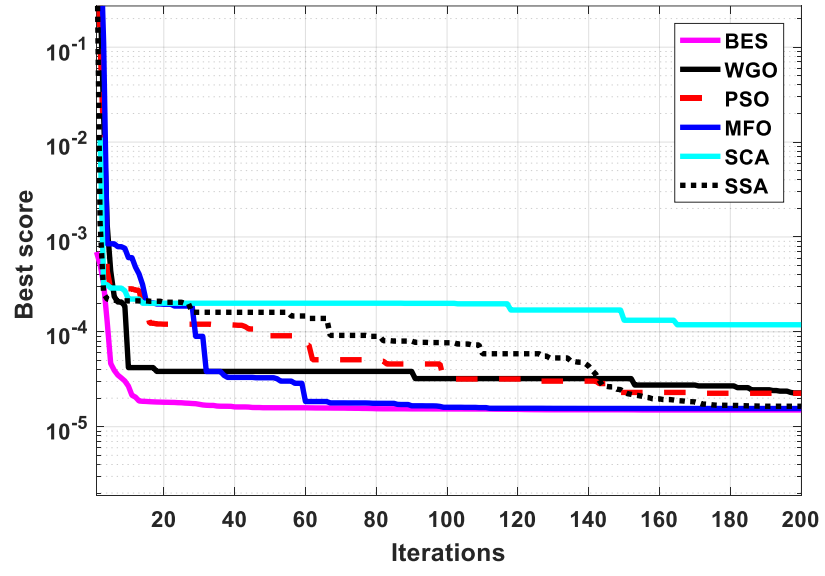

(b)

Figure 12. Convergence curves of parameter estimation using different optimizers. (a) Convergence curve of controlled device (MTDM) (b) Convergence curve of modified device (MTDM).

Further investigation of absolute current density error for both control and modified PSCs devices via using different optimization algorithms applying both TDM and MTDM, 
as explained in Figures 13 and 14, respectively. It is very easy and clear to notice that the proposed BES in both devices, and under both TDM and MTDM, has the highest coefficient of determination and the lowest mean absolute error values. This also proves the accuracy, superiority, and efficiency of the suggested BES algorithm in estimating the best parameters of both control and modified PSCs devices in both TDM and MTDM.

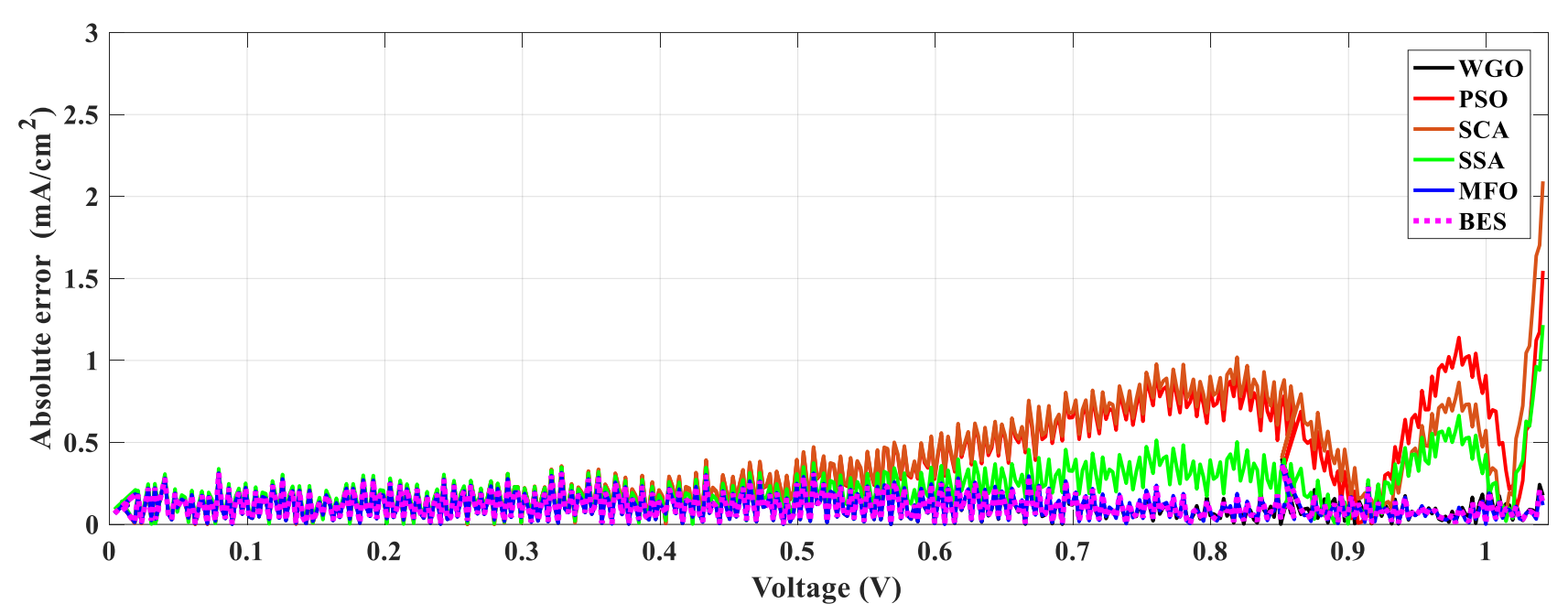

(a)

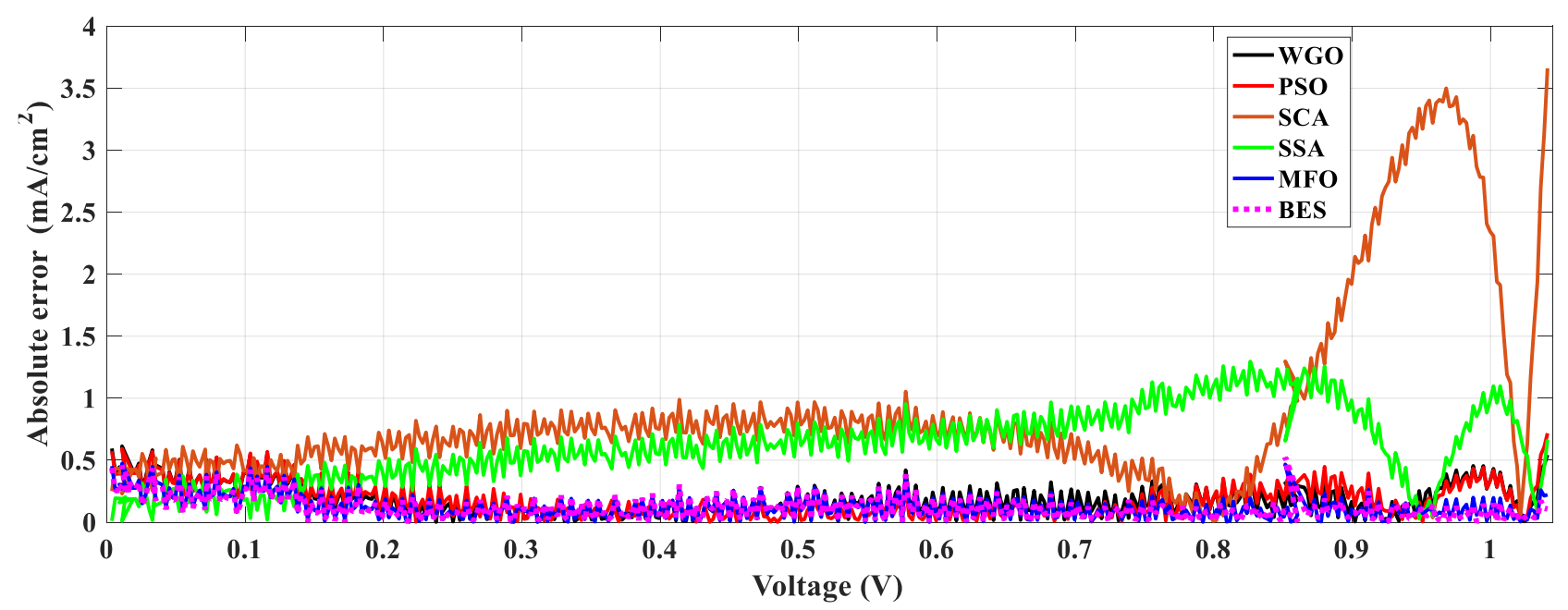

(b)

Figure 13. Absolute current density error for TDM using different optimizers. (a) Absolute current density error of control device. (b) Absolute current density error of modified device.

To prove the consistency of the proposed strategy, ANOVA test has been performed. ANOVA test results are provided in Table 9, and its related graphical ranking is illustrated in Figure 15. According to these results, the $p$-value is much lower than the F value that confirms the difference between the provided performances. From Figure 15, the BES can provide the optimal performance in terms of mean fitness. Moreover, its variations range is the lowest comparing with the other algorithms, which demonstrates its robustness. 


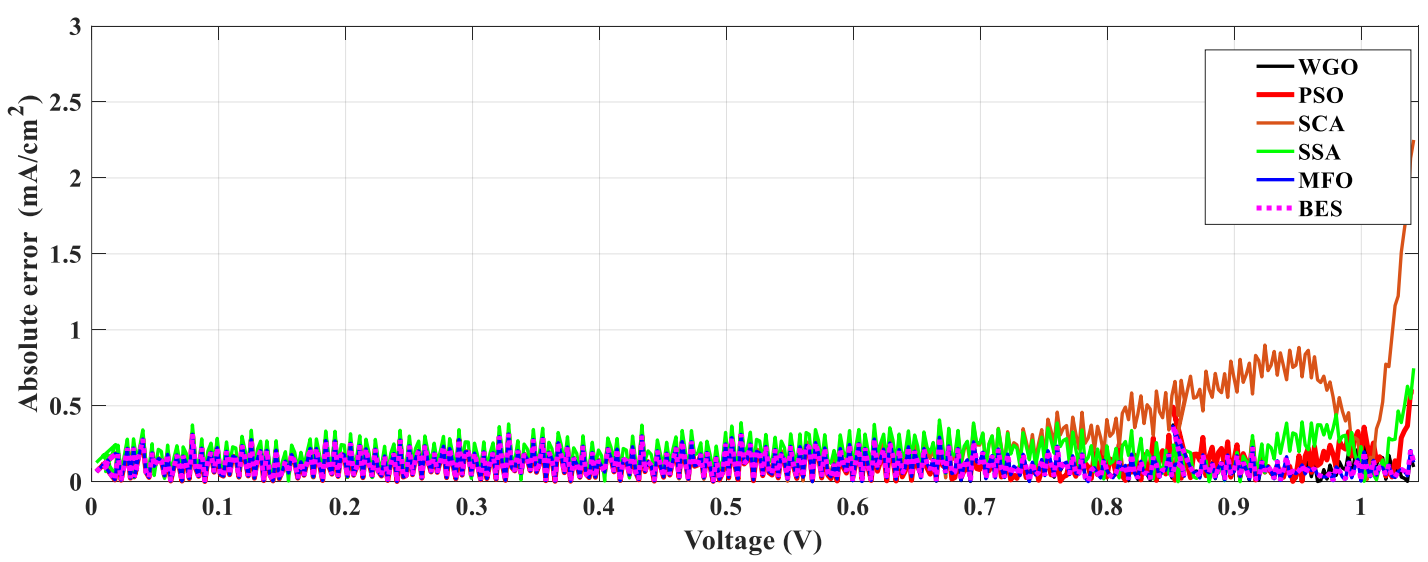

(a)

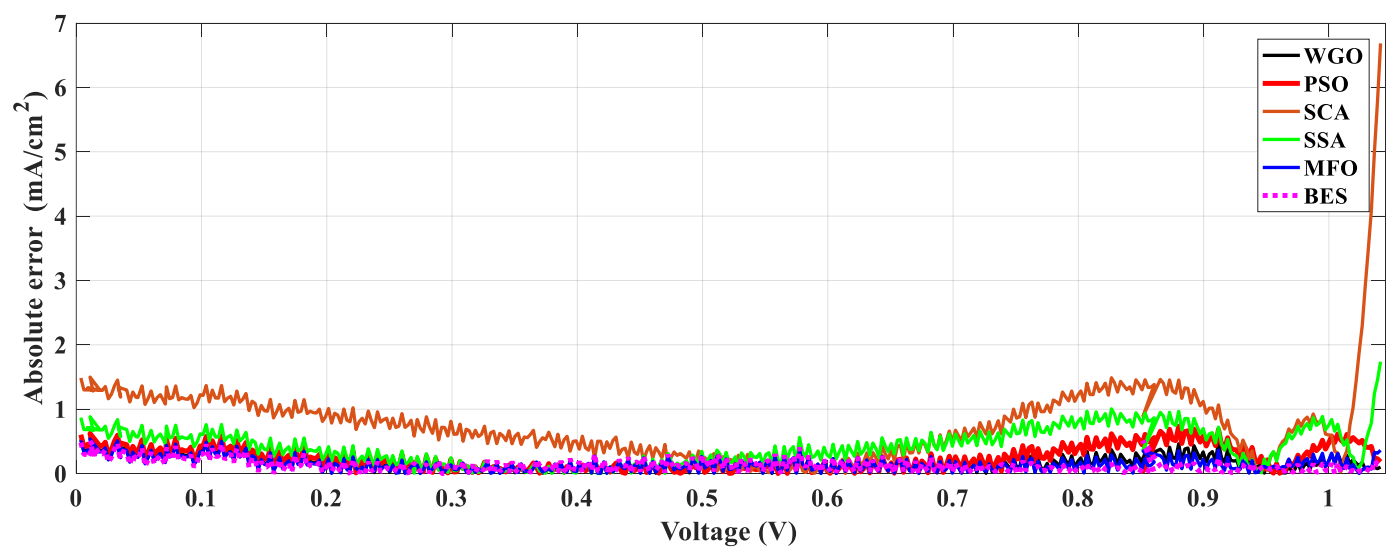

(b)

Figure 14. Absolute current density error for MTDM using different optimizers. (a) Absolute current density error of control device. (b) Absolute current density error of modified device.

Table 9. Summary of ANOVA test.

\begin{tabular}{|c|c|c|c|c|c|}
\hline Source & SS & Df & MS & $\mathbf{F}$ & $p$-Value $>$ F \\
\hline \multicolumn{6}{|c|}{ Controlled device TDM } \\
\hline Columns & $7.3739 \times 10^{-7}$ & 5 & $1.47749 \times 10^{-7}$ & 60.44 & $2.89696 \times 10^{-36}$ \\
\hline Error & $4.2455 \times 10^{-7}$ & 174 & $2.43998 \times 10^{-7}$ & & \\
\hline Total & $1.1619 \times 10^{-7}$ & 179 & & & \\
\hline \multicolumn{6}{|c|}{ Modified device TDM } \\
\hline Columns & $1.0737 \times 10^{-6}$ & 5 & $2.14785 \times 10^{-7}$ & 13.11 & $7.81786 \times 10^{-11}$ \\
\hline Error & $2.85107 \times 10^{-6}$ & 174 & $1.63855 \times 10^{-5}$ & & \\
\hline Total & $3.92486 \times 10^{-6}$ & 179 & & & \\
\hline \multicolumn{6}{|c|}{ Controlled device MTDM } \\
\hline Columns & $3.88762 \times 10^{-6}$ & 5 & $7.77524 \times 10^{-7}$ & 30.29 & $4.4604 \times 10^{-22}$ \\
\hline Error & $4.46715 \times 10^{-6}$ & 174 & $2.56733 \times 10^{-5}$ & & \\
\hline Total & $8.35477 \times 10^{-6}$ & 179 & & & \\
\hline \multicolumn{6}{|c|}{ Modified device MTDM } \\
\hline Columns & $6.93988 \times 10^{-7}$ & 5 & $1.38798 \times 10^{-7}$ & 56.2 & $1.46076 \times 10^{-34}$ \\
\hline Error & $4.2969 \times 10^{-7}$ & 174 & $2.46952 \times 10^{-5}$ & & \\
\hline Total & $1.1236 \times 10^{-6}$ & 179 & & & \\
\hline
\end{tabular}




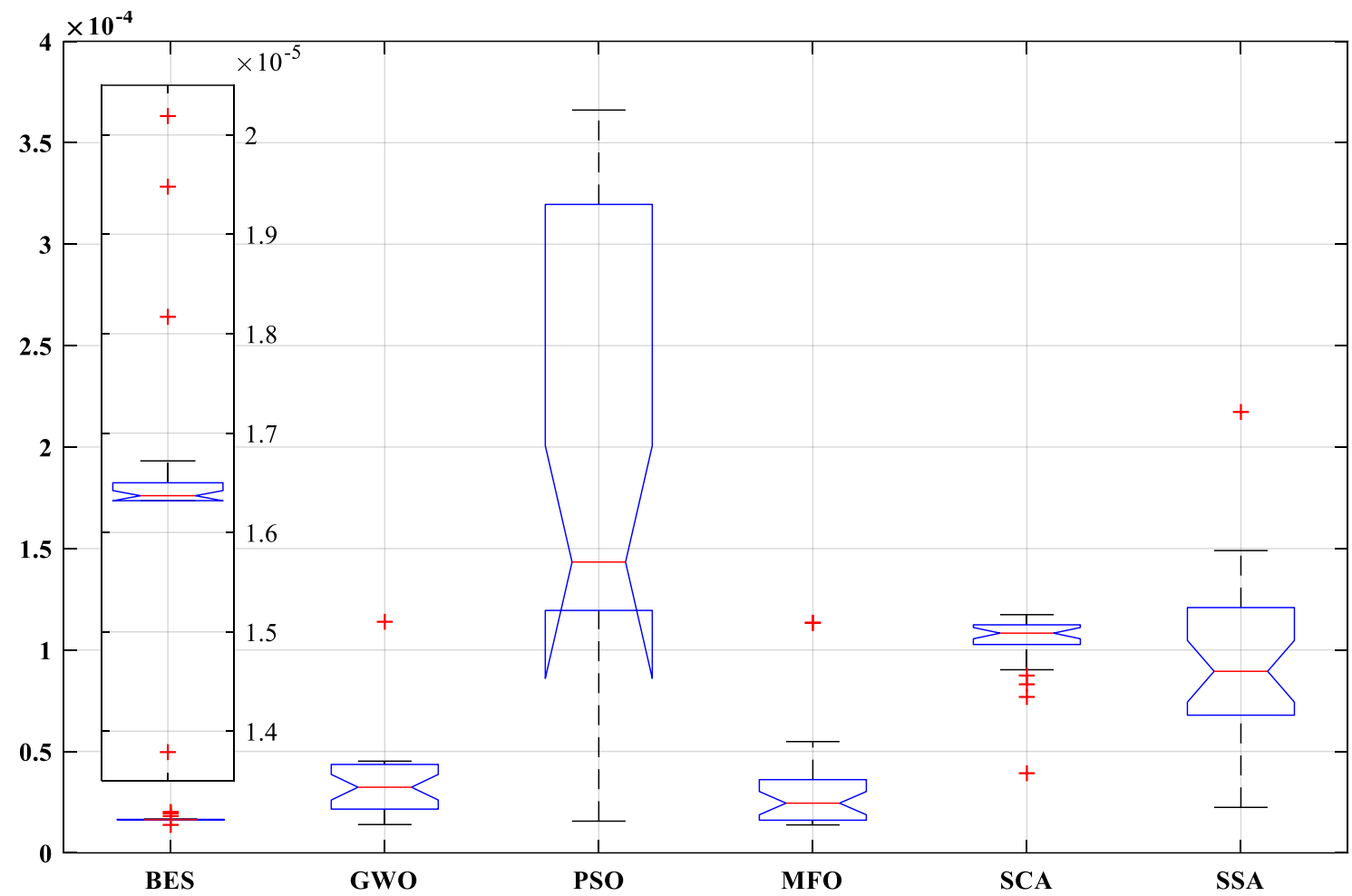

(a)

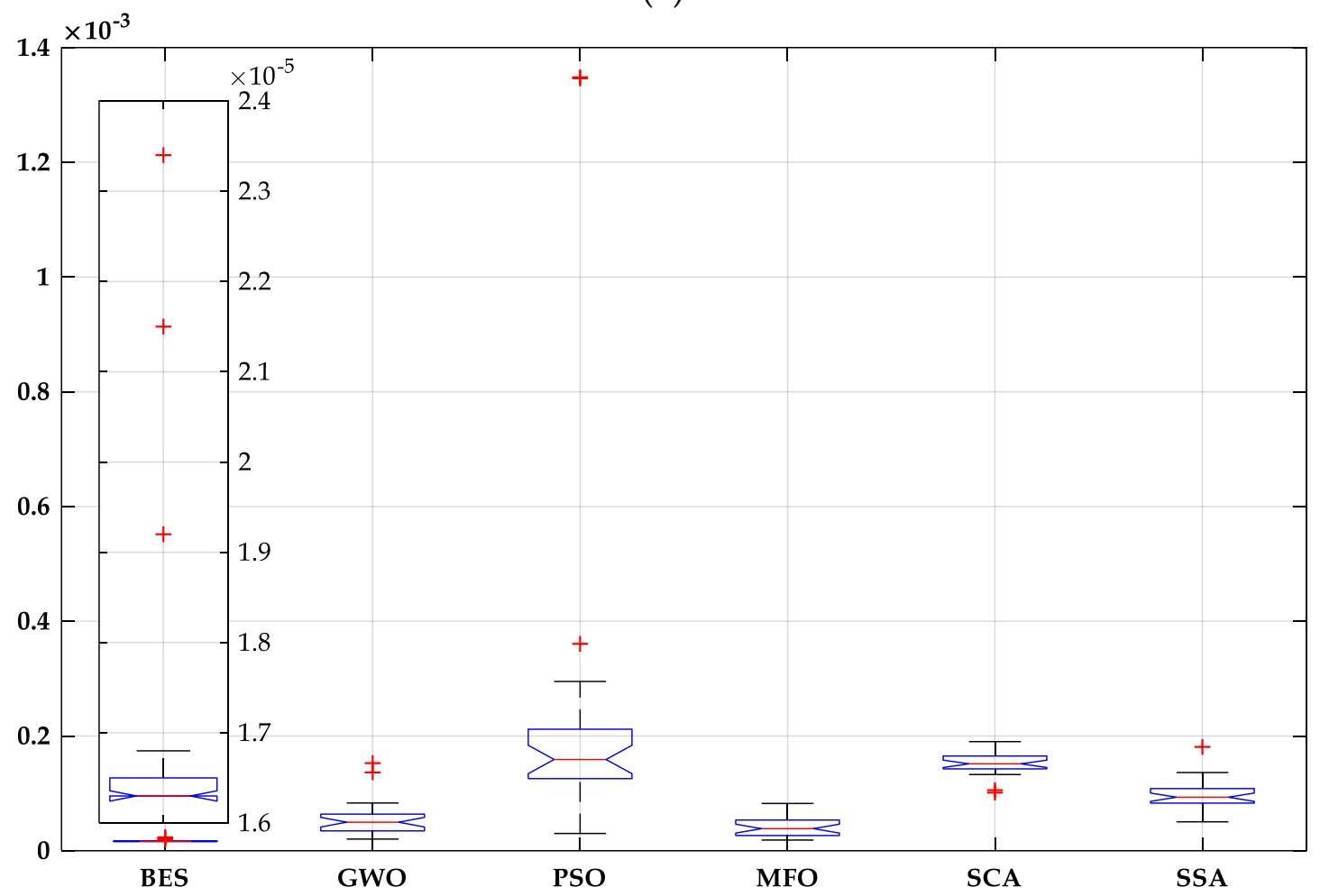

(b)

Figure 15. Cont. 


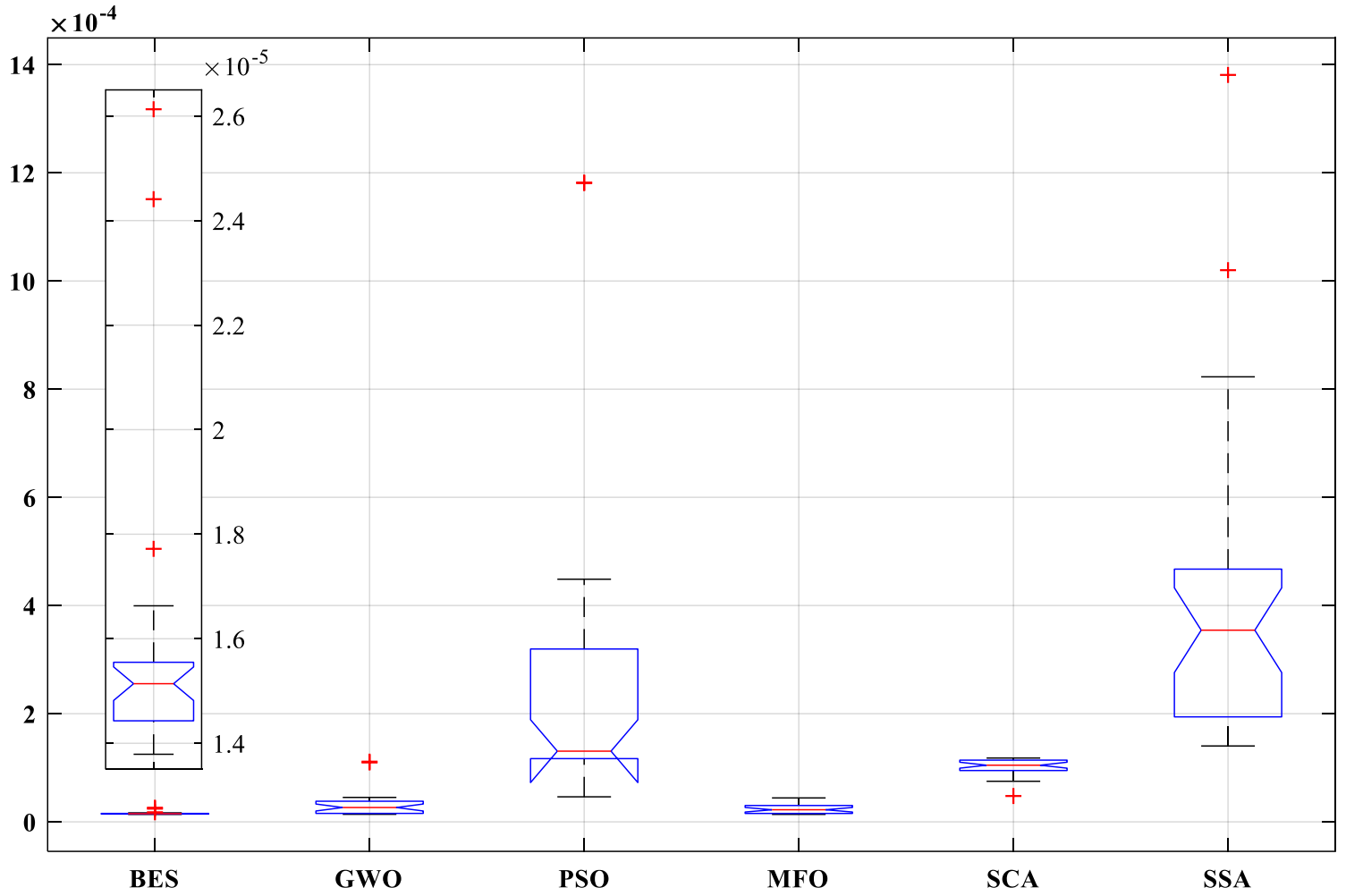

(c)

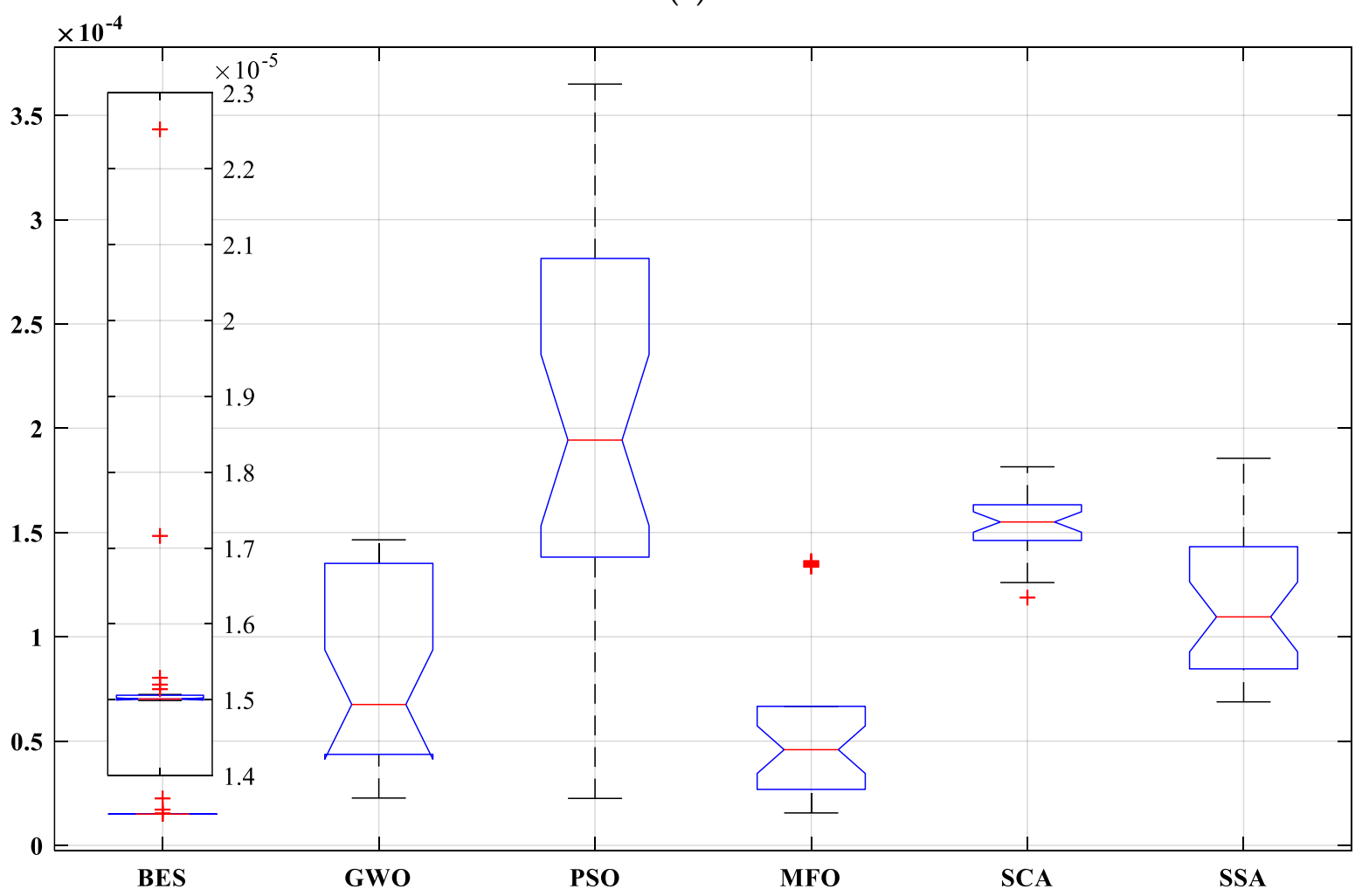

(d)

Figure 15. Observations through the independent runs based on ANOVA test. (a) Controlled device TDM- (b) Modified device TDM(c) Controlled device MTDM- (d) Modified device MTDM. 


\section{Conclusions and Future Work}

In this work, the performance enhancement of PSCs via interface engineering approach based on porphyrin insertion over the electron transporting layer is discussed. The modified devices outperform the control ones in both power conversion and stability. Electrical modeling was employed for elucidating the observed behavior. In this context, the BES algorithm was adopted for solving both TDM and MTDM electrical models and the corresponding parameters were successfully assessed. The accuracy of the BES algorithm has been authenticated using the I-V and P-V curves of the fabricated PSC devices and it has been confirmed that the main PSC parameters determined by combining the proposed simulation and the modified three-diode model efficiently fit in with the experimental characteristics at least deviation. Moreover, the proposed model permits the determination of the diode ideality factor representing the structural defects resulting from the fabrication process, which cannot be obtained experimentally. The performed analysis gives lower diode ideality factor values for the porphyrin-modified PSCs, in comparison with those of the non-modified ones. This confirms the enhanced quality of the corresponding devices, prepared following the interface engineering approach, which leads to perovskite absorbers with a low number of grain boundaries. The performed modeling revealed that the proposed MTDM operates better than TDM in representing the PSCs behavior. In addition, the BES algorithm achieves the least RMSE values in comparison with concurrent optimization approaches, as the corresponding simulation is the most efficient method for extracting the MTDM parameters. Therefore, interface engineering approaches combined with advanced modeling based on modern and efficient optimization algorithms is recommended for photovoltaic parameters estimation and device optimization in order to develop highly performing and robust PSCs.

Author Contributions: A.A.Z., A.A. and H.R.: conceptualization, methodology, software, validation, formal analysis, investigation, resources, writing-original draft preparation; A.F.: review and editing; K.G., P.F.: project administration, funding acquisition. All authors have read and agreed to the published version of the manuscript.

Funding: This research was funded by the European Regional Development Fund of the European Union and Greek national funds through the Operational Program Competitiveness, Entrepreneurship and Innovation, under the call RESEARCH-CREATE-INNOVATE (project code: T1EDK03547, MIS 5033808).

Institutional Review Board Statement: Not applicable.

Informed Consent Statement: Not applicable.

Data Availability Statement: No new data were created or analyzed in this study.

Conflicts of Interest: The authors declare no conflict of interest.

\section{References}

1. Maes, J.; Balcaen, L.; Drijvers, E.; Zhao, Q.; De Roo, J.; Vantomme, A.; Vanhaecke, F.; Geiregat, P.; Hens, Z. Light Absorption Coefficient of CsPbBr3 Perovskite Nanocrystals. J. Phys. Chem. Lett. 2018, 9, 3093-3097. [CrossRef] [PubMed]

2. Stoumpos, C.C.; Malliakas, C.D.; Kanatzidis, M.G. Semiconducting Tin and Lead Iodide Perovskites with Organic Cations: Phase Transitions, High Mobilities, and Near-Infrared Photoluminescent Properties. Inorg. Chem. 2013, 52, 9019-9038. [CrossRef]

3. Stranks, S.D.; Eperon, G.E.; Grancini, G.; Menelaou, C.; Alcocer, M.J.P.; Leijtens, T.; Herz, L.M.; Petrozza, A.; Snaith, H.J. ElectronHole Diffusion Lengths Exceeding 1 Micrometer in an Organometal Trihalide Perovskite Absorber. Science 2013, 342, 341-344. [CrossRef] [PubMed]

4. Kojima, A.; Teshima, K.; Shirai, Y.; Miyasaka, T. Organometal Halide Perovskites as Visible-Light Sensitizers for Photovoltaic Cells. J. Am. Chem. Soc. 2009, 131, 6050-6051. [CrossRef] [PubMed]

5. Liu, M.; Johnston, M.B.; Snaith, H.J. Efficient Planar Heterojunction Perovskite Solar Cells by Vapour Deposition. Nature 2013, 501, 395-398. [CrossRef]

6. NREL. Best Research-Cell Efficiency Chart. Available online: https:/ /www.nrel.gov/pv/cell-efficiency.html (accessed on 21 November 2021).

7. Liu, D.; Li, S.; Zhang, P.; Wang, Y.; Zhang, R.; Sarvari, H.; Wang, F.; Wu, J.; Wang, Z.; Chen, Z.D. Efficient Planar Heterojunction Perovskite Solar Cells with Li-doped Compact $\mathrm{TiO}_{2}$ Layer. Nano Energy 2017, 31, 462-468. [CrossRef] 
8. Roose, B.; Goödel, K.C.; Pathak, S.; Sadhanala, A.; Baena, J.P.C.; Wilts, B.D.; Snaith, H.J.; Wiesner, U.; Graätzel, M.; Steiner, U.; et al. Enhanced Efficiency and Stability of Perovskite Solar Cells Through Nd-Doping of Mesostructured $\mathrm{TiO}_{2}$. Adv. Energy Mater. 2016, 6, 1501868. [CrossRef]

9. Zhou, H.; Chen, Q.; Li, G.; Luo, S.; Song, T.-B.; Duan, H.-S.; Hong, Z.; You, J.; Liu, Y.; Yang, Y. Interface Engineering of Highly Efficient Perovskite Solar Cells. Science 2014, 345, 542-546. [CrossRef]

10. Yang, G.; Wang, C.; Lei, H.; Zheng, X.; Qin, P.; Xiong, L.; Zhao, X.; Yan, Y.; Fang, G. Interface Engineering in Planar Perovskite Solar Cells: Energy Level Alignment, Perovskite Morphology Control and High-Performance Achievement. J. Mater. Chem. A 2017, 5, 1658-1666. [CrossRef]

11. Balis, N.; Zaky, A.A.; Perganti, D.; Kaltzoglou, A.; Sygellou, L.; Katsaros, F.; Stergiopoulos, T.; Kontos, A.G.; Falaras, P. Dye Sensitization of Titania Compact Layer for Efficient and Stable Perovskite Solar Cells. ACS Appl. Energy Mater. 2018, 1, 6161-6171. [CrossRef]

12. Gkini, K.; Verykios, A.; Balis, N.; Kaltzoglou, A.; Papadakis, M.; Adamis, K.S.; Armadorou, K.-K.; Soultati, A.; Drivas, C.; Gardelis, S.; et al. Enhanced Organic and Perovskite Solar Cell Performance through Modification of the Electron-Selective Contact with a Bodipy-Porphyrin Dyad. ACS Appl. Mater. Interfaces 2020, 12, 1120-1131. [CrossRef]

13. Gkini, K.; Balis, N.; Papadakis, M.; Verykios, A.; Skoulikidou, M.; Drivas, C.; Kennou, S.; Golomb, M.; Walsh, A.; Coutsolelos, A.G.; et al. Manganese Porphyrin Interface Engineering in Perovskite Solar Cells. ACS Appl. Energy Mater. 2020, 3, 7353-7363. [CrossRef]

14. Allam, D.; Yousri, D.A.; Eteiba, M.B. Parameters extraction of the three diode model for the multi-crystalline solar cell/module using Moth-Flame Optimization Algorithm. Energy Convers. Manag. 2016, 123, 535-548. [CrossRef]

15. Abdelminaam, D.S.; Said, M.; Houssein, E.H. Turbulent flow of water-based optimization using new objective function for parameter extraction of six photovoltaic models. IEEE Access 2021, 9, 35382-35398. [CrossRef]

16. Alsattar, H.; Zaidan, A.A.A.; Zaidan, B.B. Novel meta-heuristic bald eagle search optimisation algorithm. Artif. Intell. Rev. 2020, 53, 2237-2264. [CrossRef]

17. Abate, S.Y.; Wu, W.-T.; Pola, S.; Tao, Y.-T. Compact $\mathrm{TiO}_{2}$ Films with Sandwiched Ag Nanoparticles as Electron-Collecting Layer in Planar Type Perovskite SOlar Cells: Improvement in Efficiency and Stability. RSC Adv. 2018, 8, 7847-7854. [CrossRef]

18. Sun, C.; Wu, Z.; Yip, H.-L.; Zhang, H.; Jiang, X.-F.; Xue, Q.; Hu, Z.; Hu, Z.; Shen, Y.; Wang, M.; et al. Amino- Functionalized Conjugated Polymer as an Efficient Electron Transport Layer for High-Performance Planar-Heterojunction Perovskite Solar Cells. Adv. Energy Mater. 2016, 6, 1501534. [CrossRef]

19. Yang, H.; Fan, W.; Hills-Kimball, K.; Chen, O.; Wang, L.-Q. Introducing Manganese-Doped Lead Halide Perovskite Quantum Dots: A Simple Synthesis Illustrating Optoelectronic Properties of Semiconductors. J. Chem. Educ. 2019, 96, 2300-2307. [CrossRef]

20. Tavakoli, M.M.; Saliba, M.; Yadav, P.; Holzhey, P.; Hagfeldt, A.; Zakeeruddin, S.M.; Gratzel, M. Synergistic Crystal and Interface Engineering for Efficient and Stable Perovskite Photovoltaics. Adv. Energy Mater. 2018, 9, 1802646. [CrossRef]

21. Ma, J.; Chang, J.; Lin, Z.; Guo, X.; Zhou, L.; Liu, Z.; Xi, H.; Chen, D.; Zhang, C.; Hao, Y. Elucidating the Roles of TiCl 4 and PCBM Fullerene Treatment on $\mathrm{TiO}_{2}$ Electron Transporting Layer for Highly Efficient Planar Perovskite Solar Cells. J. Phys. Chem. C 2018, 122, 1044-1053. [CrossRef]

22. Charalambidis, G.; Georgilis, E.; Panda, M.K.; Anson, C.E.; Powell, A.K.; Doyle, S.; Moss, D.; Jochum, T.; Horton, P.N.; Coles, S.J.; et al. A Switchable Self-Assembling and Disassembling Chiral System based on a Porphyrin-Substituted PhenylalaninePhenylalanine Motif. Nat. Commun. 2016, 7, 12657. [CrossRef]

23. Lazarides, T.; Delor, M.; Sazanovich, I.V.; McCormick, T.M.; Georgakaki, I.; Charalambidis, G.; Weinstein, J.A.; Coutsolelos, A.G. Photocatalytic Hydrogen Production from a Noble Metal Free System based on a Water Soluble Porphyrin Derivative and a Cobaloxime Catalyst. Chem. Commun. 2014, 50, 521-523. [CrossRef]

24. Mikroyannidis, J.A.; Charalambidis, G.; Coutsolelos, A.G.; Balraju, P.; Sharma, G.D. Novel Zinc Porphyrin with Phenylenevinylene Meso-Substituents: Synthesis and Application in Dye-Sensitized Solar Cells. J. Power Sources 2011, 196, 6622-6628. [CrossRef]

25. Stangel, C.; Daphnomili, D.; Lazarides, T.; Drev, M.; Krasovec, U.O.; Coutsolelos, A.G. Noble Metal Porphyrin Derivatives Bearing Carboxylic Groups: Synthesis, Characterization and Photophysical Study. Polyhedron 2013, 52, 1016-1023. [CrossRef]

26. Tountas, M.; Verykios, A.; Polydorou, E.; Kaltzoglou, A.; Soultati, A.; Balis, N.; Angaridis, P.A.; Papadakis, M.; Nikolaou, V.; Auras, F.; et al. Engineering of Porphyrin Molecules for Use as Effective Cathode Interfacial Modifiers in Organic Solar Cells of Enhanced Efficiency and Stability. ACS Appl. Mater. Interfaces 2018, 10, 20728-20739. [CrossRef]

27. Liang, J.; Liu, Z.; Qiu, L.; Hawash, Z.; Meng, L.; Wu, Z.; Jiang, Y.; Ono, L.K.; Qi, Y. Enhancing Optical, Electronic, Crystalline, and Morphological Properties of Cesium Lead Halide by Mn Substitution for High-Stability All-Inorganic Perovskite Solar Cells with Carbon Electrodes. Adv. Energy Mater. 2018, 8, 1800504. [CrossRef]

28. Liu, W.; Chu, L.; Liu, N.; Ma, Y.; Hu, R.; Weng, Y.; Li, H.; Zhang, J.; Li, X.; Huang, W. Efficient Perovskite Solar Cells Fabricated by Manganese Cations Incorporated in Hybrid Perovskites. J. Mater. Chem. C 2019, 7, 11943-11952. [CrossRef]

29. Jiang, X.; Gros, C.P.; Chang, Y.; Desbois, N.; Zeng, L.; Cui, Y.; Kadish, K.M. Tetracationic and Tetraanionic Manganese Porphyrins: Electrochemical and Spectroelectrochemical Characterization. Inorg. Chem. 2017, 56, 8045-8057. [CrossRef]

30. Zaky, A.A.; Christopoulos, E.; Gkini, K.; Arfanis, M.K.; Sygellou, L.; Kaltzoglou, A.; Stergiou, A.; Tagmatarchis, N.; Balis, N.; Falaras, P. Enhancing efficiency and decreasing photocatalytic degradation of perovskite solar cells using a hydrophobic copper-modified titania electron transport layer. Appl. Catal. B Environ. 2021, 284, 119714. [CrossRef] 
31. Zaky, A.A.; Ibrahim, M.N.; Rezk, H.; Christopoulos, E.; El Sehiemy, R.A.; Hristophorou, E.; Kladas, A.; Sergeant, P.; Falaras, P. Energy Efficiency Improvement of Water Pumping System Using Synchronous Reluctance Motor Fed by Perovskite Solar Cells. Int. J. Energy Res. 2020, 44, 11629-11642. [CrossRef]

32. Zaky, A.A.; Balis, N.; Gkini, K.; Athanasekou, C.; Kaltzoglou, A.; Stergiopoulos, T.; Falaras, P. Dye Engineered Perovskite Solar Cells under Accelerated Thermal Stress and Prolonged Light Exposure. ChemistrySelect 2020, 5, 4454-4462. [CrossRef]

33. Zaky, A.A.; El Sehiemy, R.A.; Rashwan, Y.I.; El Hossieni, M.A.; Gkini, K.; Kladas, A.; Falaras, P. Optimal Performance Emulation of PSCs using the Elephant Herd Algorithm Associated with Experimental Validation. Electron. Photonic Devices Syst. Sect. ECS J. Solid State Sci. Technol. 2019, 8, Q249-Q255. [CrossRef]

34. Balis, N.; Zaky, A.A.; Athanasekou, C.; Silva, A.M.; Sakellis, E.; Vasilopoulou, M.; Stergiopoulos, T.; Kontos, A.G.; Falaras, P. Investigating the Role of Reduced Graphene Oxide as a Universal Additive in Planar Perovskite Solar Cells. J. Photochem. Photobiol. A Chem. 2020, 386, 112141. [CrossRef] 